\title{
Collecting egocentric network data with visual tools: A comparative study
}

\author{
Betina Hollstein $^{1 *(\mathbb{D})}$, Tom Töpfer ${ }^{1,2}$ and Jürgen Pfeffer ${ }^{3}$ \\ ${ }^{1}$ SOCIUM - Research Center on Inequality and Social Policy, University of Bremen, Bremen, Germany \\ ${ }^{2}$ Institute for Educational Science, University of Hildesheim, Hildesheim, Germany (email: toepfert@uni-hildesheim.de) \\ ${ }^{3}$ Bavarian School of Public Policy, Technical University of Munich, Munich, Germany (email: juergen.pfeffer@hfp.tum.de) \\ *Corresponding author. Email: betina.hollstein@uni-bremen.de
}

Special Issue Editors: Brea L. Perry, Bernice A. Pescosolido, Mario L. Small, and Ann McCranie

\begin{abstract}
When collecting egocentric network data, visual representations of networks can function as a cognitive aid for depicting relationships, helping to maintain an overview of the relationships, and keeping the attention of the interviewees. Additionally, network maps can serve as a narration generator in qualitative and in mixed-methods studies. While varying visual instruments are used for collecting egocentric network data, little is known about differences among visual tools concerning the influence on the resulting network data, the usability for interviewees, and data validity. The article provides an overview of existing visually oriented tools that are used to collect egocentric networks and discusses their functions, advantages, and limitations. Then, we present results of an experimental study where we compare four different visual tools with regard to networks elicited, manageability, and the impact of follow-up questions. In order to assess the manageability of the four tools, we used the thinking aloud method. The results provide evidence that the decision in favor of a specific visual tool (structured vs. unstructured) can affect the size and composition of the elicited networks. Follow-up questions greatly affect the elicited networks and follow-up cues can level out differences among tools. Respondents tend to prefer the concentric circles tool, with some differences in preferences and manageability of tools between participants with low and those with high socioeconomic status. Finally, assets and drawbacks of the four instruments are discussed with regard to data quality and crucial aspects of the data collection process when using visual tools.
\end{abstract}

Keywords: egocentric networks; mixed methods; qualitative network analysis; data collection; network maps; data quality

\section{Introduction}

Collecting egocentric network data (Perry et al., 2018) is a task that is challenged by several obstacles. With regard to self-reported data, remembering and comparing associates can make considerable cognitive demands on memory and cognitive capacity of study participants (Marsden, 2011). As previous studies have shown, several factors can affect the accuracy of selfreports; the work in the wake of the Bernard et al. (1981) studies and the studies by Brewer (1995, 2000), Brewer et al. (2005) are especially noteworthy in this respect. ${ }^{1}$ The detailed collection of network data can be exhaustive and fatiguing for respondents and interviewers (e.g., Herz \& Petermann, 2017), possibly resulting in methodological artifacts and affecting data quality.

In this respect, visual interfaces that assist respondents seem to be promising tools. Antonucci's diagram of concentric circles (1986) is an example of such an instrument that is widely used in social support research. However, it is just recently that network researchers increasingly employ

(C) The Author(s) 2020. Published by Cambridge University Press. This is an Open Access article, distributed under the terms of the Creative Commons Attribution licence (http://creativecommons.org/licenses/by/4.0/), which permits unrestricted re-use, distribution, and reproduction in any medium, provided the original work is properly cited. 
visual tools such as network visualizations, maps, charts, sociograms, or free designs during data collection (e.g., Straus, 2002; Hollstein, 2002; Bernardi et al., 2007; Hogan et al., 2007; McCarty et al., 2007; Schönhuth et al., 2013; Ryan et al., 2014; Herz et al., 2015; Bellotti, 2016; Tubaro et al., 2016; Reyes, 2016; Dobbie et al., 2018). ${ }^{2}$ Visual tools are applied in very different ways in standardized network surveys as well as in qualitative network studies, and they can target different goals (Hogan et al., 2007; Hollstein \& Pfeffer, 2010; Bellotti, 2016). Previous studies stressed several advantages of visual collection tools: A simultaneous visual representation of network alters can serve as a cognitive aid for depicting the relationships, helping to maintain an overview of the relationships, recalling alters, and keeping the attention of the interviewees (Bellotti, 2016; Ryan et al., 2014). Additionally, network maps constitute strong narration stimuli and can serve as a narration generator, that is, as a "trigger for reflection and discussion" (Dobbie et al., 2018: 212) when used in qualitative interviews (Straus, 2002; Hollstein, 2011; Ryan et al., 2014) and mixed-methods research designs (Molina et al., 2014). Since alters are represented visually to the interviewee, the conversation can easily be (re-)directed toward certain persons, also encouraging comparisons among relationships to alters (Bernardi et al., 2007).

Other aspects of network data collection via visual tools seem to be less clear. Because the visual display can serve as a "motivational and cognitive scaffold" (Von der Lippe \& Gamper, 2017: 436), it is assumed that network maps increase the proportion of revealed alters in social networks and elicit more complete networks; however, empirical verification shows mixed results (Von der Lippe \& Gamper, 2017). ${ }^{3}$ Besides, the graphical display might improve reliability (Hogan et al., 2007) and validity of network data (Coromina \& Coenders, 2006; Von der Lippe \& Gamper, 2017), but the evidence is scarce. In these regards, the way instruments are implemented (mode, prompts) and the role of interviewers in the application of these tools can be critical. ${ }^{4}$ For instance, Dobbie et al. (2018) mentioned, when reflecting on their qualitative interviews, that they were "involved in a constant process of providing reassurance and guidance, and engaging in dialogue with the participants as they talked through their thoughts and feelings while they placed their alters" (p. 212; similarly, Ryan et al., 2014). However, systematic research on different modes of implementation of visual tools for collecting egocentric network data is scarce (Eddens \& Fagan, 2018). Additionally, most studies evaluating the use of network maps focus on specific groups, for example, gamblers (Dobbie et al., 2018), highly skilled migrants (Ryan et al., 2014), or undergraduate students (Von der Lippe \& Gamper, 2017) and use one specific type of network map, for example, standardized concentric circles (Von der Lippe \& Gamper, 2017), partly standardized concentric circles (Ryan et al., 2014), or structured but unstandardized concentric circles (Dobbie et al., 2018). Further questions concern the handling and manageability of visual tools; for example, Carrasco et al. (2008: 968) state that the simplicity of the concentric-circle sociogram makes it ideal for collecting data "in an intuitive and easy way." On the other hand, Ryan et al. (2014) observe that "the material act of visually depicting social relationships and ranking ties in degrees of closeness, makes some participants feel uncomfortable and exposed in ways that did not occur when we previously interviewed them without this tool" (9.4; similarly, Dobbie et al., 2018). As they conclude, "a visualization tool is not simply a neutral device for 'capturing' network data" (Ryan et al., 2014: 11.2). They argue that graphical tools shape social relationships into a particular format: "Its layout and design do not merely illustrate the network but influence how it is visualized by participants" (Ryan et al., 2014).

In the following, we address some of these questions related to the collection of egocentric network data by means of visual tools. We begin with a systematic overview of existing visual tools that are used to collect egocentric networks (unstructured maps, structured and standardized maps, structured but unstandardized maps, and mixed types) and discuss their functions, advantages, and limitations. Secondly, we will present the results of an experimental study where we compare four different visual tools with regard to networks elicited, manageability of tools (regarding shape and structuring elements), and how they are implemented (impact of follow-up 
questions). These tools are the diagram of concentric circles or so-called hierarchical mapping technique (Antonucci, 1986), two modifications of the funnel tool (Hollstein et al., 2013), and free network drawings. In order to assess the four tools, we used the thinking aloud method (Ericsson \& Simon, 1993) that encourages interviewees to verbalize their thought processes during the interview. In the last part, we discuss the assets and drawbacks of the four tools and crucial methodological aspects of the data collection process when using visual tools.

\section{Visual tools for egocentric network data collection: An overview}

A wide array of different visual tools to collect egocentric network data is documented in the literature. Fields of application are social support research (e.g., Wagner et al., 2018), communication research (e.g., Hepp et al., 2016), migration research (e.g., Bilecen, 2016), and life-course research (e.g., Hollstein, 2002; Bernardi et al., 2014), to name just a few. Furthermore, visual tools to support egocentric network data collection are employed not only when investigating personal networks but also in other areas, such as organizational research (Häussling, 2014) or the analysis of cultural knowledge creation (Haselmair et al., 2014).

Graphical displays in network data collection can take very different forms. Some are more standardized, as the method of concentric circles (Antonucci, 1986). On the other end of the spectrum, we find so-called "Free style drawings" that interviewees produce on their own with practically no guidelines being provided (e.g., Scheibelhofer, 2011; Reyes, 2016). Several tools employ concentric circles indicating different degrees of closeness or importance (Todd, 1980; Antonucci, 1986; Straus, 1995, Samuelsson et al., 1996; Pettigrew, 1997; Pahl \& Spencer, 2004; Hogan et al., 2007; Bilecen, 2016), using between three (e.g., Antonucci, 1986) and seven (Hersberger, 2003) circles. Some tools use sectors indicating different role relationships or life domains, such as family, friends, work, and leisure activities (e.g., Tracy \& Whittaker, 1990; Straus, 1995; Samuelsson et al., 1996; Ryan et al., 2014). In some network maps, alter-alter relationships and groups are also visualized, for example, by encircling alters (Hogan et al., 2007).

Furthermore, the implementation and handling of the instruments can take different forms (cf. Schönhuth et al., 2013). Often, the interviewees register the relationships themselves, but sometimes this is done by the interviewers. Traditionally, paper and pencil have most often been employed, while recently software solutions for a visual online and/or offline data collection have become more prominent; for example, EgoNet.QF (Hollstein \& Pfeffer, 2010), VennMaker (Gamper et al., 2012), Network Canvas (Hogan et al., 2016), or OpenEddi (Eddens \& Fagan, 2018). When combined with qualitative interviews, in some studies, the visualization is the starting point of the interview and, in other studies, the visualization is created parallel to the interview. Furthermore, while most studies use visual tools for eliciting alters, some studies elicit names by other means and then arrange names in a visual instrument (e.g., Hogan et al., 2007; Kuhns et al., 2015).

In the following, we provide a systematic overview of the different visual tools. Based on the extent of structuring and the level of standardization, we distinguish among different types of network maps: unstructured network maps, structured and standardized network maps, structured but unstandardized network maps, and partially standardized network maps (mixed types). We describe these types, illustrate application scenarios of these maps, and discuss their benefits and possible limitations. A major difference among these maps concerns the comparability of the maps and of network alters. Depending on the intended comparison (inter- and intrapersonal, cross-sectional, and longitudinal), different elements of structuring and standardization are needed.

These map layouts will also be part of our own empirical case study discussed later in this article, where we investigate how informants perceive and handle some of these features. 


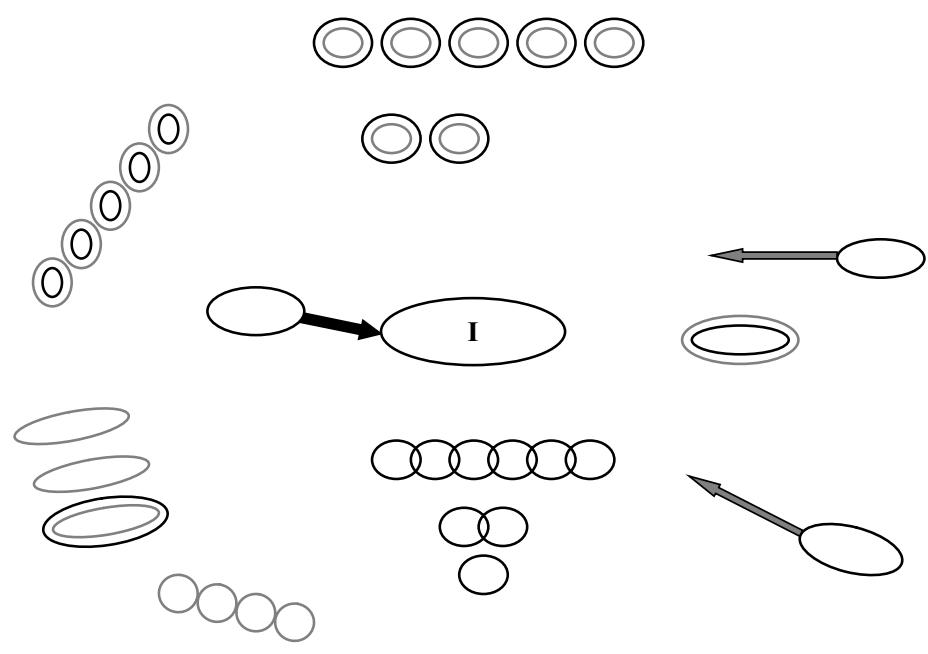

Figure 1. Reconstruction of an unstructured map from Scheibelhofer (2011: 116).

\subsection{Unstructured network maps}

Unstructured network maps are "free style drawings" (also "free designs," "hand-drawn network maps") that interviewees produce on their own with practically no guidelines being provided (e.g., Coates, 1985; McCarty et al., 2007; Hepp et al., 2016; Reyes, 2016). For instance, Scheibelhofer (2011) used such a free design in her study of the lives and the perspectives on the mobility of junior researchers from Austria who have emigrated to the U.S.A. In order to investigate the significance these migrant researchers assign to certain relationships, interviewees were asked to graphically illustrate significant persons in their private and professional lives. Participants were handed a sheet of paper with a circle in the center containing the word "I" (cf. Figure 1). The only instruction provided was to position more important persons closer to "I" and less important persons closer to the margins. ${ }^{5}$ After completing the map, the interviewees were asked to elaborate on the relationships they had noted, their meaning and significance. The instrument was kept as unstructured and flexible as possible to allow respondents to follow their own system of relevance while ensuring the least possible interference. Used in this way, the emerging map served the main purpose of generating narratives. Later, these narratives were analyzed by means of interpretative methods (Grounded Theory Methodology) in order to reconstruct different mobility types and network types of the "mobility pioneers" (Scheibelhofer, 2011). Thus, comparisons among networks were not made on the map alone. Instead, the map served as an ancillary tool to gain access to actors' cognitive representation of their networks and the meanings they attribute to social relationships.

\subsection{Structured and standardized network maps}

The so-called "hierarchical mapping technique" or "method of concentric circles" (Kahn \& Antonucci, 1980; Antonucci, 1986) is an example of structured and standardized network maps; it is widely used in psychology, gerontology, and social support research. Participants are given a sheet of paper with three concentric circles and are asked to enter on the diagram the initials of people personally important to them. The center of the sheet of paper represents the interviewee and is labeled "you" or "me" - that is, "ego" (cf. Figure 3 as an example from our own study). The space around ego is structured in three concentric circles representing differing degrees 
of emotional closeness of alters toward ego. The central element of this tool is a standardized stimulus for every circle. Participants are asked to place "people to whom you feel so close that it is hard to imagine life without them" in the innermost circle. The initials of "people who are important in your life right now" are placed in the second circle, and "people whom you haven't already mentioned, but who are close enough and important enough in your life that they should be placed in your personal network" are placed in the outer circle (Antonucci, 1986: 10). Often, additional attributes of alters and relationships are collected later in the interview, such as gender, age, place of living, role relationship, or length of relationship (e.g., Lang \& Carstensen, 1994; Hollstein, 2002; Bernardi et al., 2007). In the study of Hogan et al. (2007), who used a similar tool, participants additionally marked which alters belong to certain groups, such as voluntary associations or neighborhood, and which alters know each other.

The main advantage of tools that are structured and standardized is the comparability of network maps, both intrapersonal and interpersonal, as well as across studies. Besides, the rather unspecific stimulus "importance" does not predispose participants in a particular direction; instead, it aims directly at their individual system of relevance. Which persons are important, for what reasons, and what roles they play can be further explored in qualitative interviews (e.g., Hollstein, 2002; Pahl \& Spencer, 2004; Bernardi et al., 2007). Due to these different features, the instrument is suited to complement surveys (e.g., Wagner et al., 1999) as well as qualitative research on social networks.

\subsection{Structured but unstandardized network maps}

In contrast to unstructured maps, structured but unstandardized maps contain elements that structure the data collection process. However, the use of these elements is not defined in such detail as in standardized tools (e.g., Straus, 1995; Bellotti, 2016). An example is a tool developed by Straus (1995). Similar to Antonucci's (1986) instrument, there are concentric circles used as structuring elements to indicate varying degrees of closeness, yet the individual circles have no pre-defined distinct meaning. Figure 2 shows a digitalized network map that was originally collected with paper and pencil using the Straus instrument as part of a study on identity development of young adults (Keupp et al., 1997). Besides the circles, other structuring elements are sectors representing different life domains of ego (e.g., family, work, and leisure). These sectors also have no pre-defined meaning and can be named, placed, and sized freely by the interviewees to structure their egocentric network.

In this study, the network map was used as part of a qualitative interview. The map was created parallel to the interview (if a person is named in the interview that has not been discussed before, this person is added to the network map) and was used as a communication medium to talk about relations and networks.

In general, the structured but unstandardized form of collecting egocentric networks may combine aspects of the other two types described above: On the one hand, the network map can be used to support the interview and serves as additional narrative stimulus (narration generator). Additionally, the network map, as cognitive support, facilitates focusing on specific areas of life or groups of alters. On the other hand, the network map is also an end in itself. The additional style elements (Straus, 1995) structure the network map and, in contrast to unstructured tools, enable partial comparisons. Within one network map, ego's relations to alters can be compared in terms of the alters' assignment to circles and sectors. Different network maps of different individuals as well as of one individual over time can also be compared, although with some restrictions. However, relinquishing standardization (here, the meaning of certain circles and distances or the type of sectors) limits possibilities for comparisons, while at the same time, additional style elements increase complexity and cognitive burden for interviewees. 


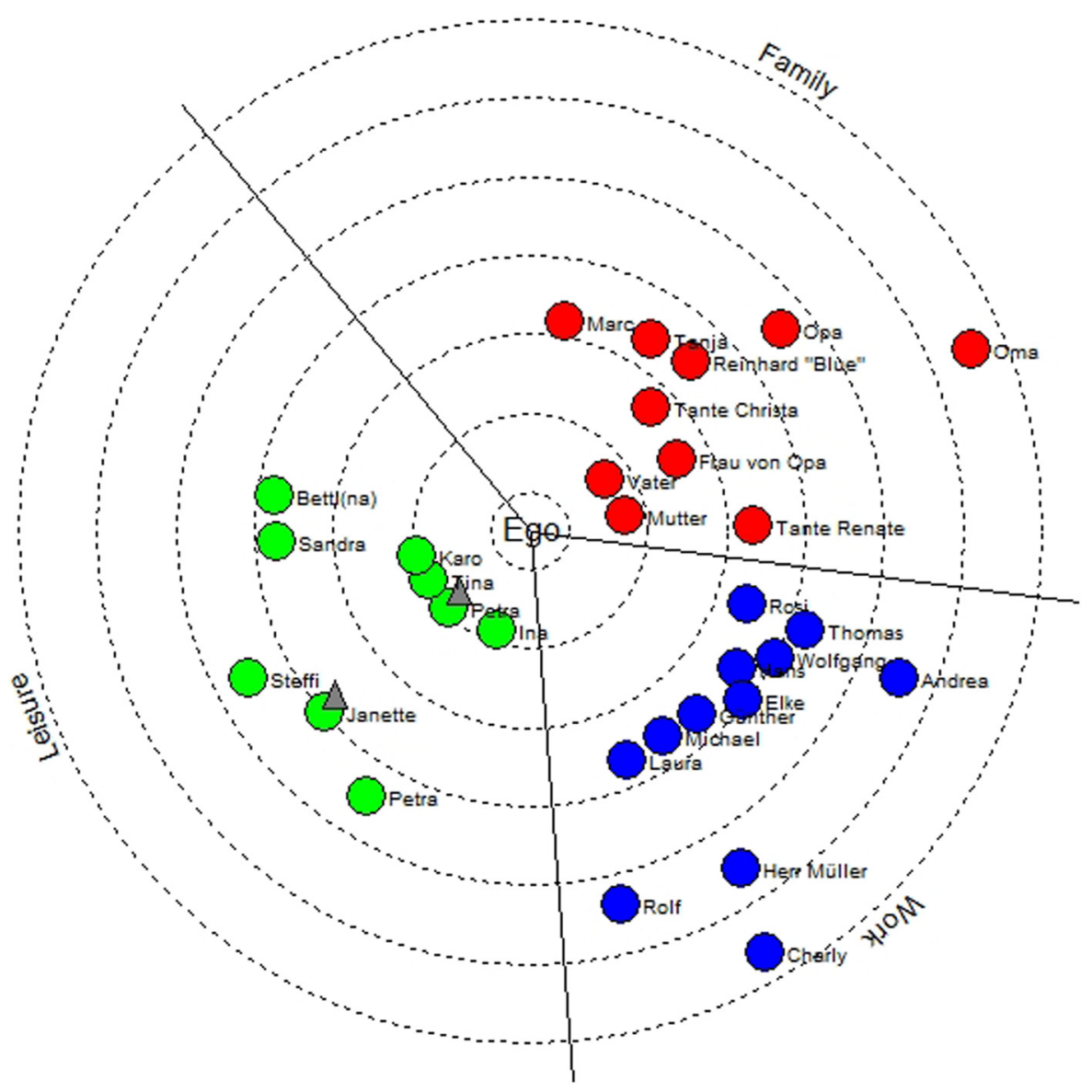

Figure 2. Structured but unstandardized tools. Straus (1995), network map created with EgoNet.QF (http://www. pfeffer.at/egonet).

\subsection{Partly standardized maps (mixed type)}

The three types just described are extremes in that they mark distinct poles of the map spectrum. In maps, for which several structuring elements (circles, segments) are used, we sometimes find mixed types, containing unstandardized as well as standardized elements, that is, instruments that are only partially standardized. For instance, the instruments presented by Samuelsson et al., 1996 and Pettigrew (1997) not only have unstandardized elements (concentric circles indicating differing degrees of closeness but without a distinct meaning for each circle) but also contain standardized elements (fixed number of sectors, fixed size of sectors, and pre-defined meaning of sectors, such as "family" and "friends"; similarly, Tracy \& Whittaker, 1990; Ryan et al., 2014). Here, comparisons between network data are also limited and depend on whether the elements in question are standardized or not.

\subsection{Comparing network data}

Comparison is a major motivation for analyzing egocentric network data. The types of network maps just described imply different opportunities for comparison among network data, including interpersonal comparisons of network maps and intrapersonal comparisons of alters (cross-sectional and longitudinal), which we will briefly discuss in the next section. At the same 
Table 1. Comparison: types of network maps

\begin{tabular}{|c|c|c|c|}
\hline & Unstructured & $\begin{array}{l}\text { Structured but } \\
\text { unstandardized }\end{array}$ & $\begin{array}{c}\text { Structured and } \\
\text { standardized }\end{array}$ \\
\hline Structuration by style elements (e.g., circles; sectors) & No & Yes & Yes \\
\hline Standardized elements (e.g., distinct meaning of circles) & No & No & Yes \\
\hline Freedom of the interviewee to exercise agency & Maximum & Limited & Limited \\
\hline \multicolumn{4}{|l|}{ Comparability } \\
\hline -Intrapersonal & No & Yes & Yes \\
\hline -Interpersonal & No & Limited & Yes \\
\hline
\end{tabular}

time, the different maps differ with respect to the leeway of the interviewees to exercise agency and to express relevancies, and the individual meaning relationships convey for informants. Table 1 depicts the major types of network maps and summarizes the respective opportunities and constraints. The table can also be read like a checklist when developing visual tools for network data collection: it refers to critical points that need to be considered (and made explicit) when network maps are applied, as well as to decisions that need to be made when preparing the data collection tool. Mixed types are not displayed. Here, comparability refers to certain elements of the visualization and the degree of their standardization.

\subsubsection{Intrapersonal comparison of alters}

Intrapersonal comparability refers to the relations between network alters. In visual representations of ego networks, this aspect mainly concerns the position of alters, that is, the comparison of distances of alters to ego as well as the positioning of alters relative to each other.

Distance to ego. The most important and often only specification for placing alters into a network map is to create different distances of alters to ego. Often, this is accomplished by means of concentric circles. If the circles (i.e., distances to ego) have a pre-defined stimulus, then every alter can be considered separately when added to the network map. If there are no distinct stimuli, then ideally every alter must be considered in relation to all other alters. In this regard, concentric circles-even if not labeled-are helpful style elements for comparing distances of alters.

Positions relative to each other. Usually, the placement of alters within the circles has no special meaning. In order to reach positional comparability of alters within a network map, participants might be tempted to compare all alters with each other. However, positioning alters on a map by incorporating the pairwise real-world distances can easily lead to frustration, since even from a computational perspective, it is impossible to perfectly map the locations of actors of a social network onto a two-dimensional space (Hennig et al., 2012). Therefore, requesting the positions of alters in relation to one another should usually be avoided. An alternative is to divide the network map into sectors (as done by Tracy \& Whittaker, 1990; Straus, 1995; Samuelsson et al., 1996; Pettigrew, 1997) which allows at least a partial systematization of the positions of alters in relation to each other. Another approach targets groups and cliques in networks and asks interviewees to encircle alters that know each other or form groups to represent these connections on the network map (Hogan et al., 2007). These procedures can be used as proxies; however, they cannot substitute for detailed collection of information on all alter-alter relationships, which is necessary when the precise structure of these relationships is the main concern (McCarty et al., 2007).

\subsubsection{Interpersonal comparability of network maps}

Interpersonal comparison of network maps describes the comparison of network maps of different interviewees. This challenge is pivotal for most studies that analyze egocentric networks 
(e.g., to study the differences of networks of men and women or different groups of migrants). To discuss these questions, a certain level of structuring of the network maps is inevitable, for example, by means of circles or sectors. Moreover, some standardization of the meaning of the style elements used is required, that is, style elements must be defined carefully. Lack of definitions increases the freedom of interpretation not only for the interviewee but also for the interviewer, which results in reduced comparability. If the focus of the study is on distances of alters to ego (e.g., with respect to their emotional closeness to ego or their relevance for certain tasks in a company; Häussling, 2014), it does not suffice to just use concentric circles and tell the informants that the map is about daily tasks or emotional closeness. Without concrete information about the specific meaning of the particular circles, it is not possible to compare different network maps (either from different people or from one person over time). ${ }^{6}$ The same is true for using sectors as style elements (Tracy \& Whittaker, 1990; Straus, 1995; Samuelsson et al., 1996; Pettigrew, 1997). Decisions must be made as to whether the number, the type (family, friends, work, etc.), or the size of the sectors should be pre-defined or can be designed freely by the interviewee.

Considerations regarding the comparability of network data are important aspects in deciding which tool might be most appropriate to use in an empirical study. Other important factors are data quality and manageability of the tools, factors we now turn to.

\section{Comparing four visual tools: An experimental study}

\subsection{Research questions}

In the following, we present the results of an experimental study where we investigate the impact of different graphical elements on the data collection process and data quality. We will focus on three major questions:

1. How do informants deal with structured and/or standardized elements of visual tools for egocentric network data collection vs. unstructured tools? Do informants react differently to elements that structure/standardize maps, such as the concentric circles in the Antonucci diagram described above? For example, do participants struggle when assigning alters to the diagram? How do they deal with the descriptions? What about the number of circles or segments? Do informants need more (or fewer) segments? On the other hand, how do participants deal with a free design tool? Which collection approach seems to be more demanding? Do such differences affect the elicited network data?

2. How does the shape or layout of visual tools affect network data? Are there differences among different visual representations of egocentric networks regarding network size, associations, and preferences by the participants, as well as the manageability of the tools?

3. Additionally, referring to studies on interviewer effects and intrusiveness (e.g., Herz \& Petermann, 2017), as well as memory organization (e.g., Brewer, 2000), we want to test the impact of cues on the activation of alters. Does the implementation of the tools (i.e., whether there are follow-up questions to the original prompt) affect the resulting network data-and if so, in what respect? Furthermore, does the type of follow-up question affect the resulting network? We are especially interested in differences between rather unspecific prompts and specific follow-up questions pointing to certain social contexts (foci; Feld, 1981), such as neighbors or work colleagues who might not be at the center of attention in the first place.

\subsection{Methods}

To answer these questions, we conducted an experimental study comparing the implementation of four different visual tools to collect egocentric network data. In order to explore the cognitive schemes and associations of the participants and the manageability of the tools (regarding shape 
and structuring elements), we used the thinking aloud method (Ericsson \& Simon, 1993) and asked participants to verbalize their thoughts during and after the data collection process.

\subsubsection{The four visual tools}

We compared the data collection process and the resulting networks using four different visual tools (treatment). In order to assess the manageability of structuring and standardizing elements vs. their absence, we compared "structured and standardized" maps with an unstructured map. To learn more about the possible impact of different visual layouts on network data and data collection process, we included three different shaped tools that represent various positions and perspectives of ego: One is the concentric-circle diagram in the adaptation of Antonucci (1986), which we described above. Another, a funnel tool (Hollstein et al., 2013), has a trapezoidal shape that represents ego's view toward the world and was motivated by the use of touchscreen computers that sit flat on the table in front of the interviewee. In contrast to Antonucci's (1986) egocentric form of visualization, in which the interviewee is asked to depict him-/herself in the center of the network map, the interviewee uses the funnel tool as the projection of his/her social surroundings from his/her physical position relative to the screen. The interviewee pulls alters that are emotionally closer nearer to the ego bar and pushes more distant alters away. ${ }^{7}$ In order to build up horizontal and vertical perspectives, we included two modifications of this tool (see Figure 3 ). The fourth tool is a free network drawing (unstructured map), consisting of a blank sheet of paper.

\subsubsection{Implementation: Initial stimulus and follow-up prompts}

We combined the visual tools with an affective name-generator stimulus that has been used in previous studies (Antonucci, 1986; adapted from Berlin Aging Study, Wagner et al., 1999). For the standardized tools, it reads as follows: "Please look at this sheet of paper. Imagine you are in the circle [or 'field' for the variants of the funnel tool] labeled 'ME': In the first circle [field], you shall put all people to whom you feel very close, so close that it would be hard to imagine life without them. In the second circle [field], you shall put all people to whom you don't feel quite so close compared to those in the first circle [field]. In the third circle [field], you shall put all people to whom you feel less close, but who are still important." For the free design, we slightly adjusted this stimulus because of the absence of pre-defined structuring elements. This stimulus reads "Please look at the blank sheet of paper. I would like you to think of all your close and important social relations. Please arrange all people to whom you feel close or that are important to you on the paper. You are free to choose how you draw your relations."

Because we wanted to exclude possible artifacts based on technological literacy, we decided to use a paper-and-pencil mode. In general, the "low tech" nature of paper-and-pencil methods is often regarded as a strength (Hogan et al., 2007: 137). For the standardized tools, we asked the respondents to write the initials of their alters on little sticky dots and put them on the map. For the free design tool, we did not give any suggestions.

In order to assess different modes of implementation, we added two follow-up questions to the original prompt: Once participants had started adding alters to their network map and when this process stalled, the first follow-up question was an unspecific cue for completeness: "Just take a moment. Is there anybody else you would like to add?" In order to examine the range of the stimulus in terms of strong and weak ties, we added a second follow-up cueing specific contexts and role relations: "Is there anyone else-for example family members, friends, colleagues, acquaintances, or neighbors?"

\subsubsection{Concurrent and retrospective thinking aloud}

In order to explore the cognitive schemes and associations of the participants and the manageability of the four tools, we used the thinking aloud method (Ericsson \& Simon, 1993), in which 

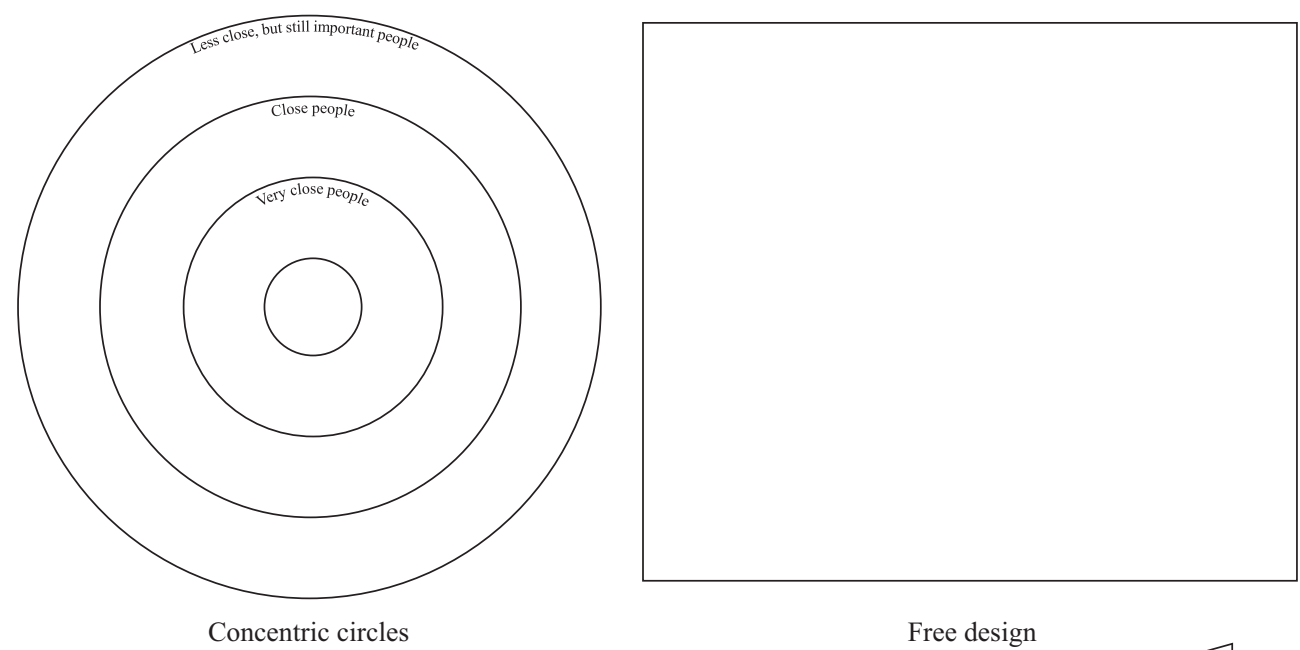

Concentric circles

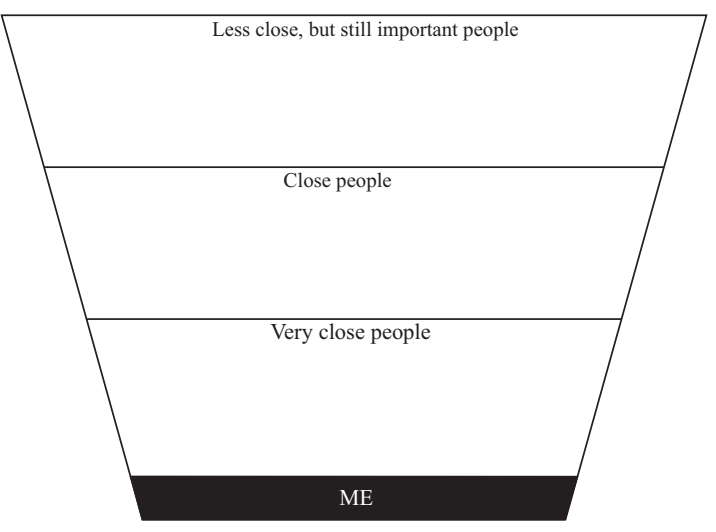

Funnel tool I

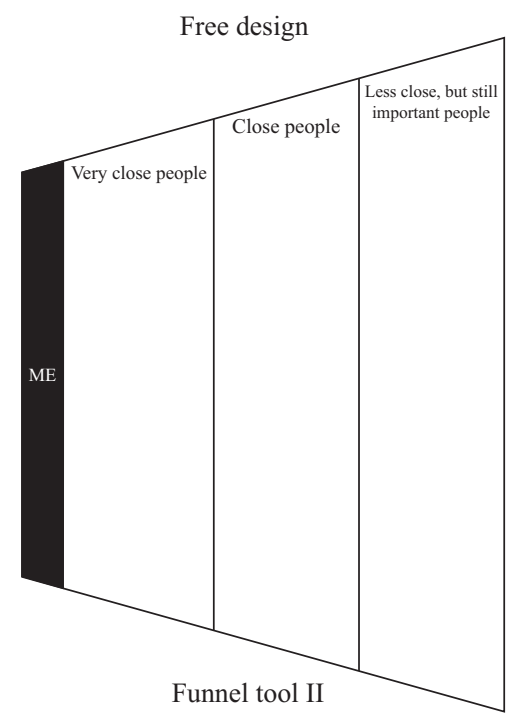

Figure 3. The four visual tools used in the experimental study. Maps are translated from German to English.

participants are asked to verbalize their thoughts during and after the process of the network data collection. In our study, we started with a warm-up exercise to familiarize participants with the thinking-aloud technique. ${ }^{8}$ After the warm-up, each participant was given one of the four tools to collect her/his personal network. Then, we started the data collection with the selected visual tool and an affective name generator, asking the respondents for concurrent thinking aloud. When participants added alters in the further interview, but did not add any comment, we asked the respondents what had triggered the naming of the alter or alters. After finishing the network data collection and the concurrent thinking aloud, we asked participants to reflect on the data collection (retrospective thinking aloud). This included questions as follows: How did participants proceed? Where were the difficulties? How did they perceive the tool? How well did they do with eliciting alters? What were their impressions in terms of the manageability of the process? How did they interpret the stimulus? ${ }^{9}$ What associations did they make to the visualization? How did they deal with the un-/structuring of the tool? This part of the interview was the key part for accessing the participants' reactions to the data collection process. After talking about the process of data collection in retrospective, we showed the participants the three other tools, explained them, asked the respondents to think about them, and finally asked which instrument they would prefer 
if they were to repeat the task a couple of weeks later. In asking respondents to reflect on network data collection in a comparative way, we avoided setting up several data collections with multiple tools for each participant as this might cause training effects or other methodical challenges. To also collect data on the network composition and alters, we added some name interpreters (role relation, gender, duration of relationship, frequency of contact, and spatial distance). As a proxy for alter-alter relationships, we also asked participants to encircle alters that belonged together in any sense, following the approach by Hogan et al. (2007).

\subsubsection{Experimental design: Randomization and stratification}

Since structural network data vary by sociodemographic characteristics, such as gender, age, and socioeconomic status (e.g., Antonucci et al., 2004; McPherson et al., 2006; Wrzus et al., 2013), and since we assumed that the manageability of the tools might also depend on sociodemographic characteristics, we decided to include participants of different sociodemographic groups in the study. We combined a purposeful sampling where characteristics of the participants (gender, age, and socioeconomic status) were varied systematically (stratification) with a randomized assignment of participants to the four treatment groups. In total, we conducted 72 semi-structured guided interviews (18 for each of the four tools; see Table 2). We decided to employ the visual tools with three age groups representing different stages of the life-course: young adults (1830 years), a group of "young old" (60-75 years), and a group of "middle-agers" (40-50 years). Socioeconomic status was operationalized by the highest educational achievement, training qualifications, and occupational status. "High" socioeconomic status typically represents persons with an academic degree working in a prestigious occupation. "Medium" status is typically a nonacademic person in a profession requiring an apprenticeship, and "low" status is typically a person with a low education certificate or no certificate and a low-skilled profession. ${ }^{10}$

\subsubsection{Data}

The field phase took place in 2014 and 2015 in the cities of Hamburg and Bremen in Germany. Six pretest interviews were conducted. In accordance with our experimental design, 72 interviews were realized. The sample consisted of 36 men and 36 women, 3 age groups (18-30 years, 4050 years, and 60-75 years) with 24 participants each, and 3 socioeconomic status groups (low, medium, and high) with 24 participants each (cf. Table 2). Each of the four visual tools was implemented with 18 participants from all 18 sociodemographic groups. Our respondents averaged 44.8 years of age; one-fourth had a migration background, ${ }^{11}$ one-third lived in a single-person household, and $44 \%$ had at least one child. The average length of each guided interview was approximately 1 hour.

\subsubsection{Interview interaction and task comprehension}

A central element of successful qualitative interviews is rapport. In order to create a comfortable atmosphere and to introduce our tasks smoothly, we started with a warm-up exercise to encourage the respondents to practice thinking aloud. Some interviewees immediately started talking and associating in response to the stimulus. Many seemed to be happy to talk a lot and stated during or after the interview that the task was "enjoyable" or "fun." A few participants started to tell sprawling stories so that we had to nudge them back to the task. On the other hand, collecting network data along with thinking aloud turned out to be quite challenging. Respondents had to interpret the stimulus, remember, compare, and order their alters, bring them on the map and, at the same time, think aloud. Besides many interviewees achieving this complex task brilliantly, a few participants hardly told us anything (during concurrent thinking aloud) and simply noted their alters, whereupon we encouraged them to think aloud while doing the data collection. Some 
Table 2. Composition of the four treatment groups.

\begin{tabular}{|c|c|c|c|c|c|c|}
\hline \multirow[b]{3}{*}{ Socioeconomic status } & \multicolumn{3}{|c|}{ Female } & \multicolumn{3}{|c|}{ Male } \\
\hline & \multicolumn{3}{|c|}{ Age } & \multicolumn{3}{|c|}{ Age } \\
\hline & $18-30$ & $40-50$ & $60-75$ & $18-30$ & $40-50$ & $60-75$ \\
\hline Low & 4 & 4 & 4 & 4 & 4 & 4 \\
\hline Medium & 4 & 4 & 4 & 4 & 4 & 4 \\
\hline High & 4 & 4 & 4 & 4 & 4 & 4 \\
\hline
\end{tabular}

participants started interacting with respect to task comprehension, expressed a need for clarification, and checked back whether they understood the task "correctly," that is, how detailed should their responses be or how should they order their relations on the map. More content-related queries addressed the stimulus' meaning. One respondent asked, "How far does 'to feel close to' go?" and then added immediately, "I'll define it myself!" Some participants felt uncomfortable giving their alters' names or initials and preferred to keep them anonymous by saying "this person," "he/she," or using role relations (e.g., "my friend"). Overall, the thinking aloud method worked well, but it should be noted that most information was obtained during the retrospective thinking aloud.

\subsubsection{Data analysis}

The concurrent and retrospective thinking aloud provided us with rich qualitative data that allowed us to capture respondents' processes in completing the task, their associations, and their intended meaning on all parts of the data collection. Combining this with the visualization of participants' networks, we were able to track the data collection process itself (including the interactions with the map and the interviewer, challenges and ambiguities in eliciting alters, and perceptions of the tools) and draw intra- and interpersonal comparisons (e.g., comparing elicitations of alters, comparing networks). Analysis of qualitative data was conducted by means of inductive qualitative content analysis. The analysis started with identifying focal interview passages on the case level. In a second step, we compared individual cases in order to systematize and categorize different patterns of perceptions, meanings, and handling of the network data collection process. All quotations from the interviews are translated from German.

Due to the experimental design combining randomized assignment to the four treatment groups (visual tools) with stratified design (sociodemographic characteristics), we were able to conduct mean comparisons ${ }^{12}$ concerning network size (cf. Table 5).

\section{Results}

\subsection{Network size and composition}

Who are the alters? Surprisingly, although the stimulus explicitly asks for close and important "persons," respondents also mentioned other actors. Several participants named groups (definite and indefinite, e.g., "members of a sports club" or "further colleagues") and institutions and organizations (e.g., "theater"). Some respondents referred to deceased persons (e.g., "my [deceased] sister ... she is still very important to me," "there are deceased persons who are important to me"); others even mentioned pets. However, it has to be noted that it is especially free network drawings that evoke networks with groups, deceased persons, or pets (cf. Table 3). Compared with the standardized tools, the free-designed networks comprise significantly more groups $\left[x_{1}=2.61\right.$ (SE 0.70), $x 2=0.85$ (SE 0.21), $p=0.006$ ]. Furthermore, animals are mentioned only with the free network drawings (cf. Table 3). 
Table 3. Types of actors and tools.

\begin{tabular}{|c|c|c|c|c|c|c|}
\hline \multirow[b]{2}{*}{ Type of actors } & \multicolumn{3}{|c|}{ Concentric } & \multicolumn{3}{|c|}{ Free network } \\
\hline & Type of prompt & circles & Funnel tool I & Funnel tool II & drawing & All tools \\
\hline \multirow[t]{2}{*}{ Single persons } & Stimulus & $11.6(5.9)$ & $12.5(7.7)$ & $12.4(6.4)$ & $7.9(6.0)$ & $11.1(6.7)$ \\
\hline & Follow-ups & $1.3(1.8)$ & $1.7(1.7)$ & $2.0(4.1)$ & $1.8(2.3)$ & $1.7(2.6)$ \\
\hline \multirow[t]{2}{*}{ Deceased persons } & Stimulus & $0.1(0.5)$ & $0.1(0.3)$ & $0.1(0.2)$ & $0.6(1.4)$ & $0.2(0.8)$ \\
\hline & Follow-ups & $0.3(0.7)$ & $0.2(0.5)$ & $0.2(0.5)$ & $0.4(1.2)$ & $0.3(0.8)$ \\
\hline \multirow[t]{2}{*}{ Groups } & Stimulus & $1.0(1.5)$ & $0.7(1.2)$ & $0.1(0.3)$ & $1.4(2.1)$ & $0.8(1.5)$ \\
\hline & Follow-ups & $0.1(0.5)$ & $0.1(0.3)$ & $0.3(0.8)$ & $1.2(2.0)$ & $0.4(1.2)$ \\
\hline \multirow[t]{2}{*}{ Animals } & Stimulus & 0 & 0 & 0 & $<0.1(0.5)$ & $<0.1(0.2)$ \\
\hline & Follow-ups & 0 & 0 & 0 & $<0.1(0.1)$ & $<0.1(0.1)$ \\
\hline Total & & $14.4(6.9)$ & $15.3(8.0)$ & $15.1(9.2)$ & $13.4(7.9)$ & $14.6(7.9)$ \\
\hline
\end{tabular}

Note: Means and standard deviations (in brackets)

Grouping alters. Condensing alters into groups is a behavior we could observe for big networks as well as for small networks, suggesting that grouping alters is not merely a consequence of fatigue. The thinking aloud method reveals several motifs for grouping alters. The first pattern represents alters that are associated together (e.g., couples, parents) or represent "natural" groups that are connected with certain activities (e.g., sports group). One respondent put it very clearly: "I feel them in groups." Groups can be important collective entities. Another motivation for grouping alters is to avoid forgetting someone. A more structural and stylistic reason is not having had enough space to list every single alter (especially for large groups and big networks). Finally, we saw respondents arguing that the process of eliciting every single alter was perceived as too time-consuming: "If I'd write down everyone, we'd sit here until tomorrow." With regard to this argument, grouping of alters can be a strategy of the interviewee to shorten the process of eliciting alters and may indicate fatigue. But, all other reasons show how cognitively challenging it is to remember "all" alters and that using groups can lighten this challenge because they can include alters that are not explicitly mentioned. Groups themselves are a specific way of clustering alters cognitively. Furthermore, groups can be very meaningful as a specific context (e.g., sociability or a special hobby), where it is not important who exactly is part of this group or how many members it has.

Network size (initial stimulus). One important question is whether the different tools differ with regard to network size; in other words, does the decision to use a specific collection approach influence the size of networks that are being collected? The number of alters varied between 3 and 48. The average network size elicited on the initial stimulus across all tools is 12.1 (SD $6.5,{ }^{13}$ range 2-34) actors $^{14}$ (nodes). The average number of alters in the three standardized tools is 12.9 (SD 6.2), compared to 9.9 (SD 6.9) in the free design (see Figure 4).

Comparison of means tests shows no significant differences in network size among the three standardized tools as well as according to age and gender (see Table 5). Looking at the standardized tools and the three segments ("circle" or "field") representing different degrees of closeness, on average 4.8 (SD 2.6) alters are set "very close," 4.1 alters (SD 2.7) are "close," and 4.1 (SD 3.5) alters are classified as "less close." This is in line with previous research showing that the first and second circles are mostly reserved for strong ties, that is, family and friends (Kahn \& Antonucci, 1980; Wagner et al., 1999), partners and family members are on average closer than acquaintances, neighbors, and colleagues. 


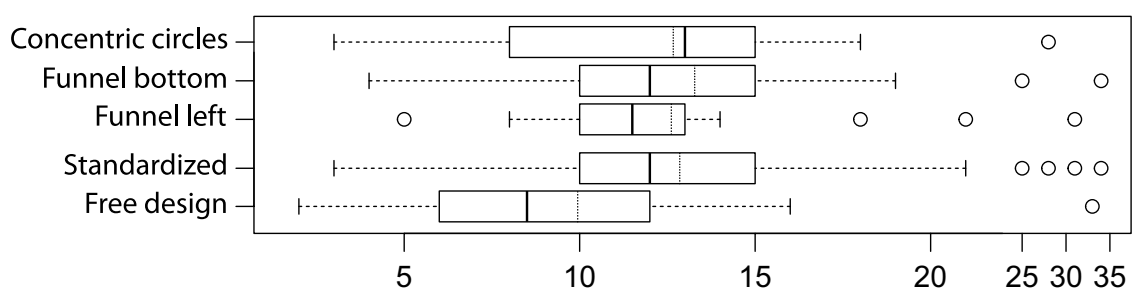

Figure 4. Boxplots network size by tool (initial stimulus only). Boxplots with median (bold lines) and mean (dotted lines) for four types of network tools as well as for three standardized tools combined.

Table 4. Network size by tool (including follow-ups).

\begin{tabular}{lccccc}
\hline & Concentric circles & Funnel tool I & Funnel tool II & Free network drawing & All tools \\
\hline Stimulus & $12.7(5.7)$ & $13.3(7.3)$ & $12.6(5.9)$ & $9.9(6.9)$ & $12.1(6.5)$ \\
\hline Follow-up 1 & $0.8(1.4)$ & $0.7(1.0)$ & $0.9(1.3)$ & $1.2(1.6)$ & $0.9(1.3)$ \\
\hline Follow-up 2 & $0.9(1.5)$ & $1.3(1.3)$ & $1.6(3.9)$ & $2.3(3.0)$ & $1.5(2.6)$ \\
\hdashline Total & $14.4(6.9)$ & $15.3(8.0)$ & $15.1(9.2)$ & $13.4(7.9)$ & $14.6(7.9)$ \\
\hline
\end{tabular}

Note: Means and standard deviations (in brackets).

Implementation of follow-up questions. Implementing two different follow-up questions, we investigated whether different kinds of cues have an impact on data collection with regard to the activation of important ties and data validity.

In sum, $62.5 \%$ of the respondents named further alters after follow-up prompts, or to put it differently, about one-sixth of alters were added to the networks after follow-up questions (see Table 4).

After the first (unspecific) follow-up question (“[...], is there anybody else you would like to add?") two out of five participants (44.4\%) named additional alters, on average 0.9 (SD 1.3, range $0-6)$ alters. Half of the alters named after the first follow-up question are family members (45\%), about $25 \%$ are friends.

After the second follow-up question, suggesting different contexts of relationship ("Is there anyone else, for example, family members, friends, colleagues, acquaintances, or neighbors?") almost half of the respondents (47.2\%) named additional alters, on average 1.5 (SD 2.6, range 016). Respondents elicited family ties in $29 \%$, but more than half of all neighbors (56\%) and about $17 \%$ of all acquaintances and $13 \%$ of all colleagues were named after the second follow-up. ${ }^{15}$

In what way do the follow-up prompts affect network size? Are there differences in this regard concerning the four tools? After follow-up prompts, the average network size for the total data collection rises from 12.1 to 14.6 (SD 7.9). The average network size for the three standardized tools rises from 12.9 to 14.9 (SD 7.9), and for the free network drawing from 9.9 to 13.4 (SD 7.9) (see Table 4). Interestingly, differences between the standardized tools and the free design tool regarding the number of alters decreased significantly during the course of data collection following the two additional prompts (see Figure 5). The unstructured tool benefited more from prompting compared with the standardized tools (see Table 4). In general, the second follow-up question that addresses specific foci induced more alters than the first one, but the proportion was less pronounced for the standardized tools compared with the free design. For the free design, the second follow-up induced twice as many alters than the first follow-up prompt. One-third of the alters named after the second follow-up question are neighbors, whereas the first follow-up question primarily induced family (37\%) and friends (30\%). In general, the free design induced nearly as many family members and friends after the stimulus as the standardized tools (72\% for the free design compared to $79 \%$ for the standardized tools). For all tools, the two followup questions lead to a slight decrease in the proportion of family and friends, but a little more 


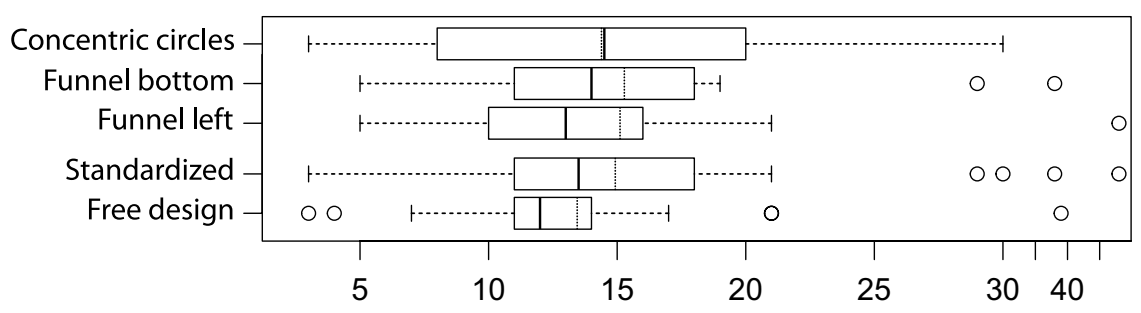

Figure 5. Boxplots network size by tool (including follow-up 1 and 2). Boxplots with median (bold lines) and mean (dotted lines) for four types of network tools as well as for three standardized tools combined.

Table 5. Differences of means in network sizes for subsets.

\begin{tabular}{|c|c|c|c|c|c|c|c|}
\hline Variable & Value 1 & $\overline{x_{1}}$ & Value 2 & $\overline{x_{2}}$ & $\overline{x_{1}}-\overline{x_{2}}$ & $p$-values & \\
\hline Standardized & Yes & $12.85(0.85)$ & No & $9.94(1.63)$ & $2.91(1.83)$ & $0.021^{*}$ & \\
\hline$(N=54 / 18)$ & & $14.93(1.08)$ & & $13.44(1.87)$ & $1.48(2.16)$ & 0.259 & $\downarrow$ \\
\hline Age & $40-50$ & $13.79(1.50)$ & $18-30$ & $10.96(1.18)$ & $2.83(1.91)$ & 0.226 & \\
\hline$(N=24 / 24)$ & & $17.62(1.97)$ & & $12.58(1.28)$ & $5.04(2.35)$ & $0.059^{\dagger}$ & $\uparrow$ \\
\hline Status & High & $14.96(1.29)$ & Low & $9.38(0.70)$ & $5.58(1.47)$ & $0.001^{*}$ & \\
\hline$(N=24 / 24)$ & & $17.50(1.35)$ & & $11.12(0.84)$ & $6.38(1.59)$ & $<0.001^{\ddagger}$ & \\
\hline Sex & Male & $12.47(1.16)$ & Female & $11.78(1.01)$ & $0.69(1.53)$ & 0.955 & \\
\hline$(N=36 / 36)$ & & $14.89(1.50)$ & & $14.22(1.12)$ & $0.67(1.88)$ & 0.572 & \\
\hline
\end{tabular}

Note: Differences of means in network sizes for subsets based on values 1 and 2 of a variable. First lines without follow-up questions, second lines with follow-up questions. Standard errors of means and of differences in brackets. $p$-value levels for Wilcoxon test: ${ }^{\dagger}=0.1,{ }^{*}=0.05,{ }^{\S}=0.01,,^{\ddagger}=0.001$.

pronounced for the free design (66\% family and friends after the second follow-up for the free design compared to $74 \%$ for the standardized tools).

For the standardized tools, we can also detect effects of the follow-ups with regard to the degree of closeness. The first follow-up prompt generated 0.2 (SD 0.5) "very close," 0.2 (SD 0.5) "close," and 0.5 (SD 1.0) "less close" alters, while the second follow-up generated 0.2 (SD 1.0) "very close," 0.3 (SD 1.0) "close," and 0.7 (SD 1.3) "less close" alters. Proportionally, 92\% of the very close alters, as well as $87 \%$ of the close alters and $77 \%$ of the less close alters, were recalled after the initial stimulus. Thus, the two follow-up prompts induced especially "less close" alters, but also elicited a remarkable number of "close" and "very close" ties.

Table 5 shows the difference of means tests for subsets of the data based on tools, age groups, status, and gender, without as well as with follow-up questions. We see no significant differences in network size with regard to the tools after the follow-up questions. Concerning age, we see the networks of the youngest participants (18-30 years) having 12.6 (SD 6.3) alters. This is smaller than the networks of the "middle-agers" (40-50 years) who reported 17.6 (SD 9.6) alters on average. Besides age, respondents with high socioeconomic status have significantly bigger networks (17.5, SD 6.6) than low-status respondents (11.1, SD 4.1). Networks of men (14.9, SD 9.0) and women (14.2, SD 6.7) remain relatively similar in size after the follow-up questions. In sum, the results regarding differences in network size among sociodemographic groups are in line with general findings on egocentric networks (e.g., Antonucci et al., 2004; McPherson et al., 2006; Wrzus et al., 2013). However, the study revealed interesting results with respect to follow-up questions and their impact on network size. The size difference that we can observe when using standardized vs. free design tools gets smaller and loses statistical significance after the follow-up questions. The opposite effect can be observed for differences resulting from age. There, the differences get larger and become significant. For gender, the follow-up questions do not create any differences and, for status, the differences seem highly significant, independent of the interview protocol. 
Understanding the cues and impact of follow-up questions. The thinking aloud technique reveals additional information on the impact of follow-up questions: Some participants use a followup question to make sure that they have understood the question correctly (e.g., "Are deceased persons allowed?"; "Do you mean spatially close or emotionally?") and to then include alters who-from the perspective of the participants-might not belong to the group of interest for the research project, for example, deceased persons ("there are deceased people, of course, who are important to me")or persons with whom there is not much contact currently ("but who are important to me"). It is especially the dynamic of relationships that makes it difficult for some participants to classify the relationships according to closeness. Often, the prompt seems to function as a memory aid to include important alters, for example, with regard to close friends, and also to complete specific foci. In one case, it was even the spouse who was named later in the interview ("I forgot my wife, she is of course above all"). The second follow-up, especially, activates contexts and foci which might not have been at the center of attention in the first place, such as neighbors or colleagues ("ah, yes, my neighbors!"). In some cases, we got the impression that the prompt supports desirability bias. This seems especially the case for relatives. For instance, one participant enumerated three nephews because "... I don't see [them] very often, but they are family." On the other hand, drawing attention back to an already activated foci such as family might help in "completing" the alters of this life sphere. Sometimes, the follow-up evokes reasoning or even justifications as to why no more alters are included ("it is only a small circle of people").

\subsection{Visual tools: Associations, preferences, and manageability}

After the participants had finished the network data collection, we involved them in a retrospective thinking aloud and encouraged them to reflect on the data collection process and the visual tools. We asked how they perceived the shape of the tool they had worked with Section 4.2.1 and the handling of certain structuring elements, such as size and number of segments (Section 4.2.3). Afterward, we presented all available tools to ask them about which tool they thought would be most suitable for the task that they had just accomplished (Section 4.2.2).

\subsubsection{Associations regarding the shape of the tools}

Concentric circles. Several respondents perceived the concentric circles as esthetic, using terms such as "coherent" or "harmonious", and utilized relevant metaphors such as "circles around me" or "circle of friends." However, there are also ambivalent associations related to the ego-centered position, such as "I am no egocentric" or "well, I am the middle of my universe." One participant justified the ego-centered position with "As it is about me, that is totally all right. [laughs]. One has to, that's the starting point." Another participant problematized the ambivalence of the egocentered position of ego:

P.: That simplifies the task, umm, but ...that reflects, that reflects, that doesn't reflect

...that doesn't represent my understanding of our relations [...] I am no egocentric.

I.: So, you are not in the middle?

P.: (...) It is swarming. (...) It is no static thing, but one image that changes continuously. And I am certainly not in the middle. Umm, well I am one of those and there was no order. Well, umm, the perspective is right. That is from my perspective, that's my perception, but umm, otherwise umm ...

I.: You would arrange yourself differently in regard to the other persons?

P.: Yes, exactly. And above all, these persons I have cited have relations with each other.

Funnel tool I. Associations with the funnel tool I are quite different. Some participants speak of a "bucket," "a bowl," or a "funnel" (!). Others include themselves in the description ("me as 
the basis"). Some participants stress the vertical alignment ("upside-down pyramid," "hierarchy of needs," and "static"), and some informants consider the shape as very hierarchical ("up and down"). One middle-aged woman, referring to an up-and-down association of that tool, clarifies "being down" not as a negative association but more as a sort of fundament:

\section{I.: How does it feel being arranged under these three segments?}

P.: That I am at the bottom? No, that's all right. Because it's me. That is my position. That is me. And, I mean, can one, one can't replace me, because I have a clear standpoint and this is my environment. I don't feel because I stand at the bottom ... Okay, one can say, I have to be at the top and then it goes down. No, but it should build up!

Some participants also argued along the lines that in fact motivated the invention of the tool, that is, translating emotional distances into spatial distances ("near" and "far").

Funnel tool II. Regarding funnel tool II, participants reported associations similar to those mentioned for funnel tool I. Additionally, they referred to horizontal dimensions, speaking of a "visual field," a "beam of light," "structured like a table," "skewed," "trapezoidal," "someone being beside me," "like you write from left to right," and "left is the side next to the heart." One older male participant with low status outlined the different perspectives of this tool as follows: "Hmm, ... I can practically peek, stare into the distance. Let my mind wander and, ah, can sort, that's important [...] Because I am not in the middle, but stand outside and can look on my social contacts."

Free design/free network drawing. Quite different from participants' views of the three standardized instruments were the associations and handling of the free network drawing (an example is depicted in Figure 6). Three aspects were most interesting for us: First, the hurdle of the blank sheet of paper. Some people had difficulties and asked for guidance ("shall I draw, or what?"), others just went forward. Interestingly, some of them did it similarly to the way the circles are structured ("I do it in circles"). Typically, the first decision was whether participants should include themselves in the drawing or not. Each decision had different implications for the representation: Most of the participants who included themselves in the drawing had a relational perspective and tried to mark distances between ego and alter and also among alters. In contrast, others just made a list as a sort of overview. Secondly, we were surprised by the diversity and complexity of the free designs: Several informants produced lists by just naming people without any (obvious) order. Some grouped alters according to spatial and/or social distance among alters but without ego-alter relations. Some registered ego-alter relations, that is, distances between alter and ego. Some informants produced more complex network drawings, such as concentric circles. Third, many free designs were lists, which seem to be the option with the lowest threshold. This might have been encouraged by the stimulus "arrange" instead of "draw."

\subsubsection{Which visual tools do participants prefer?}

In the final part of the interview, we showed the participants the other three tools, explained them, and asked which tool they would prefer if they were asked to repeat the task. Would they take the same tool they were now familiar with, or would other tools be more appealing to them? Table 6 depicts the results. The boldfaced cells reflect those participants who preferred the tool they used during data collection; all other cells indicate deviating choices.

Somewhat differently from our first assumption, only half of the participants preferred the instrument they had just used during data collection (33 out of 72). In addition, there was a clear tendency for the concentric circles being preferred most: half of the respondents preferred the circles over the other instruments. Not only did the concentric circles have the highest proportion of "stayers" ( $2 / 3$ of the participants who had worked with this tool compared to only $1 / 3$ "stayers" in the groups who had employed other tools), they also drew most of the "movers", that is, participants who preferred different tools from the one used during data collection and who voted 
Table 6. Given tool and preferred tool.

\begin{tabular}{|c|c|c|c|c|c|}
\hline Tool\preference & Concentric circles & Funnel tool I & Funnel tool II & Free network drawing & Total \\
\hline Concentric circles & 12 & 6 & 9 & 9 & 36 \\
\hline Funnel tool I & 2 & 7 & 1 & 0 & 10 \\
\hline Funnel tool II & 2 & 1 & 6 & 1 & 10 \\
\hline Free design & 2 & 4 & 2 & 8 & 16 \\
\hline Total & 18 & 18 & 18 & 18 & 72 \\
\hline
\end{tabular}

Note: boldfaced numbers represent participants preferring the tool they used for data collection.
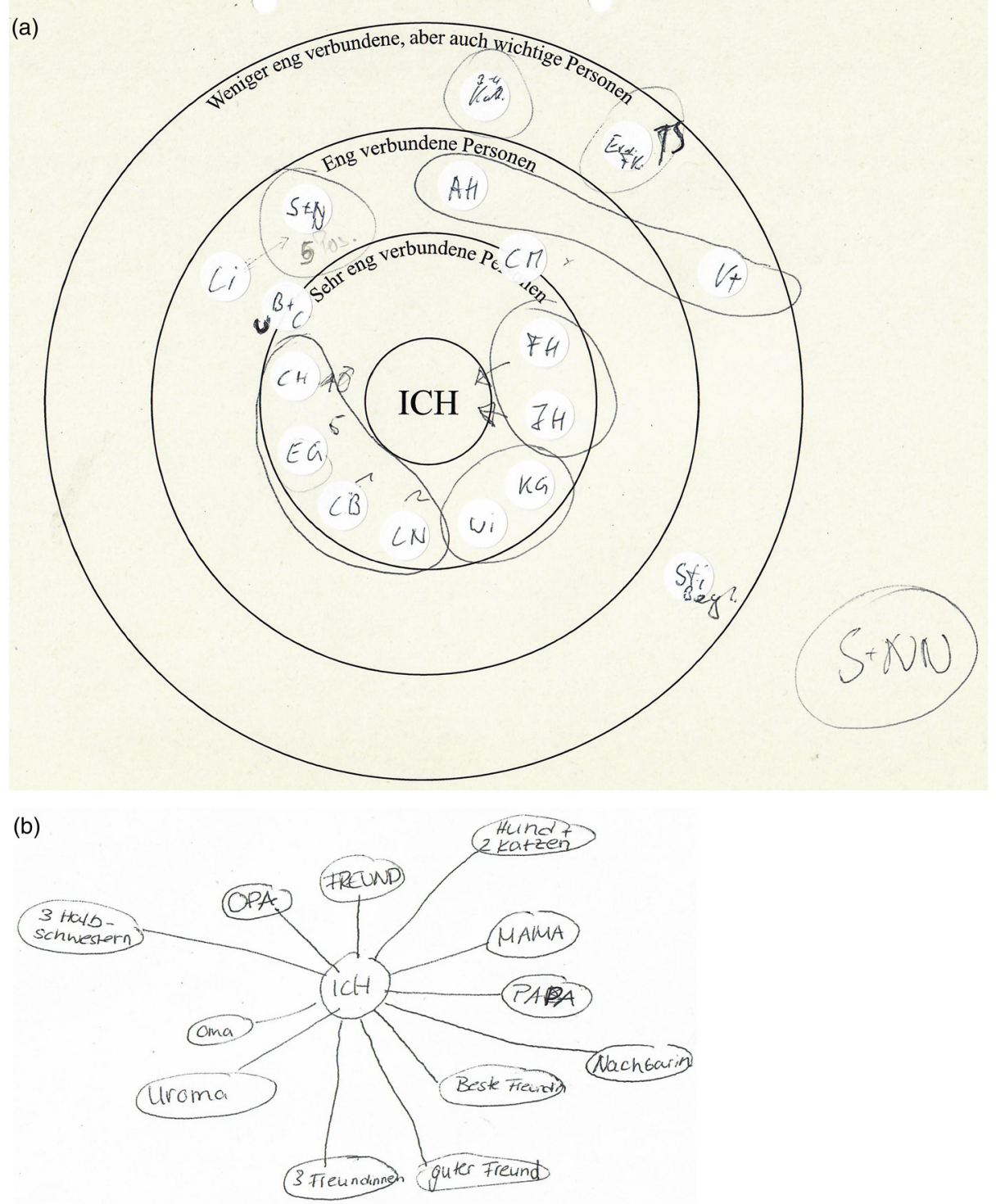

Figure 6. Examples: Structured and standardized tool (concentric circles) and unstructured tool (free network drawing). 
for the concentric circles-which can be considered as a strong indicator for the "appeal" of this instrument. This is also in line with the more favorable and less ambivalent associations regarding these tools, presented in the last section. In contrast, the other three tools are less favored.

There is also a slight but interesting tendency concerning socioeconomic status: more than half of the participants with middle (15) or high socioeconomic status (13) preferred the concentric circles, compared to only a third (8) of the respondents with low status. In addition, there is a slight tendency for participants with low status to prefer a funnel tool $(11$ compared to 6 with middle status and only 3 with high status). Or, put differently, six participants of low or middle status "moving" to one of the funnel tools and preferring this over the instrument used during data collection, compared to five belonging to the low-status group. On the other hand, the free design got the most votes from the high-status group (8) compared to only three from the middle-status group and five from the low-status group.

\subsubsection{Perceptions and manageability of structuring elements}

Finally, we looked at how participants put alters in the standardized segments and how they dealt with the free design, respectively. The comments of the participants shed further light on the cognitive processes involved in the task.

Categorizing into circles/segments. As the comments indicate, categorizing alters into the standardized diagrams was not always easy for the participants. Notably, it was especially participants from middle- and high-status groups who claimed to find it "difficult" to enter their alters into the three segments or disliked doing so. One participant (male, middle age, and high status) explained his dislike of the idea of segments representing a hierarchical order, as follows:

(...) because it's simply no, (...) it is no hierarchical relation or that what I do, would contradict the task. Umm, because, because, ... the people that I fill in here [the map], with them I do not represent hierarchical, I don't figure a hierarchical relation to them, but simply an emotional or a sort of closeness. And that is something, something different. I would know now [breathes heavily] There would be people, that were hierarchically superior, whom I could record. Yes. Such as superiors or, or ... with colleagues it would be difficult, because they have to be on the same level... So, it would have to be important superior persons, such do exist. They exist, but all the others would, could not be recorded by a hierarchical logic.

Some participants put alters on the lines between circles or (in the funnel tools) segments, indicating degrees of closeness beyond the categories offered in the diagrams. For example, one respondent hesitated to put his girlfriend in the innermost circle because they had begun dating just recently. Some respondents claimed that they would prefer gradual ("soft") transitions between the categories or that they preferred having a tool without "separating lines" ("these lines are bothering me"). A reason given by some was that they did not want to set the close people apart from each other. Others perceived their relationships as very dynamic. As one participant (middle age, high status) put it,

Well, that the three categories, yes they are default, which is difficult, umm because there is, this classification can't be done so categorically because relationships... first, because the relationships permanently change and second, because the boundaries are fluent. (...) in order to complete it [the map], I'd have to set considerably more dots and allocate sort of arrows with tendencies. Umm, where do they move to right now? Are they going to become closer, do they become more distant? Something like this.

Size of segments. As we have learned, the size of the segments indicates that there is more space for less close alters compared to close alters. In other words, the space for very close persons is 
rather limited. Here, we got different reactions: One young woman of the middle-status group commented: "Actually, it should be the other way around: because, very close persons, the field should be bigger than with less close. Because with regard to closer people you can tell more, and you have more. With less close persons who you know you remember fewer names, therefore, the field is perhaps a bit too big." For other respondents, the third segment (i.e., circle in this case) for the less close persons could have been bigger: "Umm, it was a bit difficult, for reasons of simplicity I merged persons into groups-and if I would have been more detailed, I would have needed more space" (man, middle age, and high status). Interestingly, the different sizes of the segments seem to be more obvious with the funnel tool. The respondents recognized that the shape of the tool and the size of the segments suggest a certain normative understanding of network compositions, that is, that people tend to have more less close alters than very close alters.

Number of segments and shades. The number of segments was perceived in different ways. Some respondents seemed to be forced to put alters in all segments. Asked whether she would have liked more or fewer segments, a young woman of the middle-status group responded, "No, this is subdivided just right ... to clearly arrange [my alters]. Because I don't know that many people." Here, she related the number of segments to the size of her network. Other respondents related the number of segments to the categorization process mentioned above: A middle-aged man of the high-status group replied: "Umm, it would have been easier if there would have been a scale, say, from zero to ten - and I would have assigned values to the persons, then it would have been probably more differentiated and it would not have been that categorical, but rather gradual. Umm, this would describe the relationships like I perceive them, would describe them better." One respondent imagined an additional segment being appropriate for "loose contacts (...) acquaintances you've never seen real before (...) catchword Facebook friend." Another respondent, working with the concentric circles, even allocated a group of alters beyond the circles and by doing so created a fourth category. It was a group of distant relatives living in Africa. The respondent mentioned them when talking about other relatives that he had allocated in the third circle: "Two brothers-in-law and four nieces and nephews [I.: Okay] Uhum, well, there are of course brothersin-law and nieces and nephews abroad. With them I, I should [I: You can add them if you want]. They are here, outside."

Free designs. The previously discussed structured and standardized tools appear to limit the freedom to act but at the same time offer orientation. On the other hand, the free design not only provides no orientation but also calls for a certain amount of creativity. As one elderly woman from the low-status group expressed it: "Here [with the funnel tool] you have the scale already provided. And, here [the free design] I would have to do it by myself. This would have been rather difficult for me, I believe." Another participant who had worked with the free design suggested that a categorization "might have been helpful as a starting point, but afterward, one would begin to add something, write something, ... would like to add subdivisions" (middle age, middle status). Another middle-aged man from the high-status group who had used the concentric circles replied enthusiastically when asked whether it would have been helpful to just get a blank sheet of paper: "Yes, yes, then I would have thought what characterizes the relationships. And I would have reflected on how I can display it graphically. And therefore, ... yes, I believe this would have been easier."

\section{Discussion}

In this study, we compared four visually based data collection tools for egocentric networks with regard to the elicited networks (size and composition), the manageability of the tools (regarding shape and structuring elements), and associations and preferences of the interviewees. In addition, we studied different ways of implementation (impact of follow-up questions). In general, participants preferred the concentric circles over other instruments but with notable exceptions for 
low-status (tendency toward funnel tool) and high-status groups (tendency toward free design). The satisfaction with the concentric circles is expressed in predominantly positive associations that relate the shape to metaphors for social relations and embeddedness (e.g., circle of friends). Although the satisfaction with the concentric circles seemed to be higher for most of our participants, this did not seem to affect the size and composition of the elicited networks. Within our sample, we found significant differences in network size when comparing standardized with free design tools. When follow-up prompts were used, these differences got smaller and lost statistical significance. The unstructured tool seemed to benefit more from prompting, compared to the structured tools, especially from the second follow-up question, or as a reviewer put it "structure helps recall." We could observe the opposite effect with age groups-the difference between the bigger networks of 40-50-year-old participants compared to younger participants increased further and became significant.

We used two different follow-up prompts: An unspecified prompt asking if anybody else should be added, and a follow-up question pointing to certain social contexts (family, friends, colleagues, acquaintances, and neighbors). Surprisingly, nearly two-thirds of the respondents named further alters after the follow-up cues. Follow-up questions also substantially increased the size of the networks: On average, the elicited network grew by $20 \%$ when implementing the follow-up questions. This increase in size was also accompanied by a slight change in the composition of the networks: The two follow-up prompts induced more "less close" alters than others but still elicited a remarkable amount of "close" and "very close" ties. The second follow-up prompt elicited somewhat more "less close" ties than the unspecific first one. Here, it was especially neighbors who were added disproportionately more often to the network. However, the second follow-up cue also induced a substantial amount of "close" and "very close" ties, especially family. Interestingly, the proportion of family and friends was nearly the same for the standardized tools and the free design. In general, the free design induced nearly as many family members and friends after the stimulus as the standardized tools. For all tools, the two follow-up questions led to a slight decrease in the proportion of family and friends.

The thinking aloud method helped us to understand how participants interpreted the task and why they added network members after the follow-up prompts. In several cases, the follow-up question functioned as a memory aid for adding network members who seemed to be important but had been forgotten. In these cases, the follow-up prompts supported completing the network. Sometimes the follow-up question stimulated queries by the participants about the interpretation of the question and whether they had understood it correctly. The second follow-up cue, especially, also activated contexts and foci which might be important but at first were not at the center of attention, such as neighbors, colleagues, or other acquaintances. In a few other cases, the followup prompts seemed to force participants to add names, or at least to justify why they did not add any more names (social desirability). Taken together, these aspects support the standardization and normalization of the elicited networks. The follow-up cues can open up opportunities for participants to reflect on their important network members and to ensure they had understood the interviewer's question. Together with the quantitative results, the thinking aloud provides further evidence of the sensitivity of network data collection and the crucial role of prompting and wording. Furthermore, it offers explanations for the interviewer's effects on network size that has been documented in earlier studies (e.g., Herz \& Petermann, 2017).

With regard to network size, it should be noted that not only individual actors but also groups, deceased persons, and animals were elicited by the tools, a result that we found especially surprising because interviewers had explicitly asked for "persons" (similarly, Straus, 2002; Hollstein, 2002). If non-humans are not relevant for a study, these entities may not affect network parameters because they can easily be removed from the data set. This is not possible for groups. Here, the interviews document how cognitively challenging it is to remember "all" alters and that using groups can lighten this challenge. The thinking aloud revealed that grouping alters can be due to 
fatigue, especially when large groups and big networks are involved. Besides, grouping can be a way of clustering alters cognitively, something we coined "natural groups" (similarly, Brashears \& Quintane, 2015). However, it was especially with free designs that groups were named. Free network drawings comprise significantly more groups than the standardized tools, a result which might partly account for the differences in network size among the tools after the initial stimulus.

In general, we did not find indications for differences in interviewee fatigue among the four tools. The thinking aloud method documented that the visual tools were considered as an interesting and stimulating task, providing further evidence that the graphical representation of the network engaged participants and helped sustain their attention (Bellotti, 2016; Ryan et al., 2014). However, we did not include treatment with just a verbal stimulus and so we cannot compare visual tools against non-visual tools (cf. Von der Lippe \& Gamper, 2017).

Our results also support the thesis that affective name generators are especially suitable for eliciting strong ties (Marsden \& Campbell, 1984) and less suitable for eliciting weak ties, at least when implemented without further follow-up questions that explicitly address certain contexts and foci. One might assume that the fact that the outer segments of the standardized tools are larger in size than the inner ones might encourage participants to name more "less close" ties. Although this interpretation was in fact expressed by some participants, it did not lead to differences in network size. On the contrary, along the three degrees of closeness, we found an almost equal distribution of alters, with a tendency toward more "very close" alters (also Antonucci et al., 2004). It seems that the stimulus for the third segment addressing "importance" and "closeness" (by asking for "less close" persons) does not encourage respondents to systematically name many weak ties.

Structuring elements of the standardized tools (shape of the tools, size, and the number of segments, ${ }^{16}$ distinct stimulus for every segment) not only provide opportunities for intra- and interpersonal comparisons but also provide orientation and guidance for many interviewees. On the other hand, some participants in our study expressed difficulties or dislike regarding the hierarchical ordering of their network members. Difficulties especially arose because of the dynamic nature of relationships, for example, when a close relationship had just started or when it was expected to change. Some participants found having only three levels of relationships as too limiting and preferred gradations over distinct categories. In contrast to structured tools, free designs enable creative latitude for individual network representations, but, as the interviews document, these instruments can also entail difficulties for some participants due to the missing structured orientation. Based on our stratified sample covering a broad range of sociodemographic groups ${ }^{17}$, our results suggest that these differences correspond to social status and educational attainment. It was especially participants with higher socioeconomic status (better education, higher professional status) who liked to work with the free design, whereas participants of middle and lower social status rather preferred structured tools. Similarly, Reyes (2016) started her study with creative professionals with concentric circles but had to switch to free designs to better engage with her participants. However, these are tendencies that must be solidified and perhaps differentiated on the basis of larger samples.

Besides these differences regarding the target populations, some tools might be better suited for certain research questions and analyses than for others. In the second part of this article, we systematized the potentials for inter- and intrapersonal comparisons of network maps facilitated by different types of maps. With regard to the four visual tools compared in the experimental study, recognizing the limitations in the two-dimensional space mentioned above (cf. Section 2.5), the unstructured map as well as the concentric circles provide more freedom to map distances meaningfully compared to the funnel tools. ${ }^{18}$ Researchers who are interested in the structure of a network, in network composition, or characteristics of dyads that require collecting name interpreter data of individuals (such as closeness or frequency of contact) might prefer one of the structured tools, where condensing alters into groups does not appear as frequently as with the free design tool. At any rate, researchers have to decide how to deal with groups. One option is to follow 
up by explicitly asking about group members who meet the criteria in question (e.g., "Please tell me which members of this group you consider 'less close, but still important."'). As the interviews suggest, condensing alters sometimes indicates fatigue, especially in large networks. If this should be avoided, one might want to limit the number of network members in advance. In other cases, groups are a specific way of clustering alters cognitively, something we called "natural groups" (similarly, Brashears \& Quintane, 2015). Besides, groups can indicate a meaningful context (e.g., for sociability or a special hobby), where it is not important who exactly is part of this group or how many members it has (Hollstein, 2002). If the researcher is interested in such cognitive representations, and the individual meaning the groups and social entities have for the informants (e.g., when studying network orientations, perceptions of embeddedness, and dynamics of relationships; e.g., Ryan \& D’Angelo, 2018), one might either complement a structured map with qualitative interviewing or make use of a free design within a qualitative setting (cf. Hollstein, 2011). In contrast to the standardized tools, free designs do not preset specific relational aspects (closeness, importance, etc.), thus allowing privileged access to network representations and broader concepts of embeddedness. Free designs also encourage interviewees to add dead people, objects (cf. Hepp et al., 2016), and groups. On the other hand, free designs can entail difficulties for some participants due to the lack of structure. Another downside is the limited intra- and interpersonal comparability of alter positions in free designs (cf. Section 2.5). Follow-up prompts seem to have an especially large impact on free designs, thus making at least the results on network size and composition comparable to those collected with structured instruments.

The results concerning follow-up questions imply that these prompts should be implemented with great care. The results also point to the importance of thorough interviewer training and the need for interviewers to stick to their field manual. Haphazard use of follow-up questions by only a portion of interviewers can have dramatic effects on research outcomes. Interviewers "should be made aware of the aims, scope, and limitations of network data" (Herz \& Petermann, 2017: 81) but also of the implications of their interventions. This implies having a clear (and common) understanding by all interviewers about a set of critical questions, such as "which follow-up questions are asked when," "how are groups and non-human actors handled?," "how to handle fatigue," "how to protect privacy and yet avoid concealment," ${ }^{19}$ or "how to handle creative use of the tool."

\section{Conclusions}

Social scientists in different fields increasingly employ visual tools to collect egocentric network data. Visual maps engage participants and serve as cognitive aids, helping in recalling alters and maintaining an overview of relationships. Additionally, network maps constitute strong narration stimuli and can serve as a narration generator when used in a qualitative interview and in mixedmethods research designs. Our experimental study provides evidence that the decision in favor of a specific visual tool (structured vs. unstructured) can affect the size and composition of the elicited networks. We found that follow-up questions greatly affect the elicited networks and that follow-up cues can level out differences among tools. Respondents tend to prefer the concentric circles tool, with some differences in preferences and manageability of tools between participants with low and those with high socioeconomic status, depending on the degree of structuration and the shape of the tool (in structured instruments).

Future research should further investigate these aspects. For instance, there might be cultural differences when it comes to "ego-centralization." Not everybody might view him-/herself as being in the center of the circles. In our study, we found these concerns, especially in the low-status group. The sample of our study was stratified according to gender, age, and socioeconomic status but consisted of white Westerners only. Studies that systematically compare the handling of tools and perceptions of networks among different milieus or between individualistic and collectivistic 
cultures, and perhaps also with regard to personality traits, might be enlightening. Furthermore, in an era of digitalization, different modes for implementing visual tools, such as digital instruments or online applications, are continually being developed (e.g., Hollstein et al., 2013; Fagan \& Eddens, 2015; Hogan et al., 2016). Looking ahead, different means and applications for collecting visual-based ego network data should be compared systematically with regard to manageability for different target groups (e.g., Gamper et al., 2012).

Further research should also systematically investigate the differences between network data collection with and without visual tools (e.g., McCarty et al., 2007; Von der Lippe \& Gamper, 2017). Structured visual tools are assumed to enable and facilitate comparisons between alters regarding their relationship to ego. ${ }^{20}$ By contrast, for some respondents in our study, it is exactly the fact that they have to compare their network members with each other that seems to cause uneasiness (similarly, Ryan et al., 2014). The discomfort might even be heightened because these comparisons (similarities, differences) are visualized and thus in a way objectivized. For example, it might be relatively easy to talk about a problematic relationship one has with a relative, but having to put it on a map and visually document, it could be burdensome. Furthermore, in such comparative studies, visual tools should be included for which, on the one hand, names are elicited as part of the visual task and, on the other hand, alters are arranged on a visual map but are elicited by other means (Hogan et al., 2007; Hollstein et al., 2013; Kuhns et al., 2015). Those comparisons should pay special attention to differences regarding fatigue and should include measures of efficiency. ${ }^{21}$

Finally, some difficulties respondents encountered while reflecting on the data collection process point to a general challenge in network data collection. Some participants were reluctant to allocate certain alters to a distinct category due to the dynamic nature of the relationships. These dynamic aspects of relationships and how they can be more adequately addressed in data collection are areas that are certainly worth further investigation. An interesting aspect is the recent research into so-called "dormant" ties (e.g., Levin et al., 2011). While a network map is just a snapshot, longitudinal studies, for example, combined with narrations or thinking aloud methods might shed further light on these relational dynamics and processes.

\footnotetext{
Acknowledgments. The University of Hamburg and the University of Bremen supported this research. Student assistants: Helene Windolph, Nils Baudisch, Julia McFadden, and Anna Sophie Röthe. We thank the anonymous reviewers and the editors of this special issue for their helpful comments.
}

Conflict of interest. None.

\section{Notes}

1 For example, Brewer (2000) documents extensive forgetting of associates in free recall tasks, especially concerning less close ties (Brewer, 2000) and less salient or less specific ties (Bell et al., 2007). Confer also Marsden (2011).

2 In the literature, different terms are used synonymously for visual network collection tools or instruments. In this article, we use the term maps to refer to all sorts of visual representations of personal networks. For "structured tools," we also use the term diagram.

3 Von der Lippe \& Gamper (2017) did not find differences in network size between networks collected with a visual or with a grid condition (in a condition with a pre-set maximum number of 20 alters), but when allowed to move beyond the maximum, students in the visual condition were "motivated to enter more alters than in the questionnaire" (p. 435).

4 Recent studies report considerable interviewer effects on network size (e.g., Herz \& Petermann, 2017), providing further evidence of the sensitivity of network data collection.

5 Other free network drawings are simply based on a blank sheet of paper, in order to avoid any influence on how ego-alter relations are visualized (e.g. Reyes, 2016).

6 For example, a change of an alter's position over time might be due to a change in closeness, due to a change in the esthetic perception of the map, or due to a training effect. Only the first indicates a change in the relationship. Without additional information, it is not possible to evaluate the nature of the change.

7 The associations ("near" and "far") also work on a piece of paper sitting on a table but applying the same tool to a regular (non-touch screen) computer/monitor setup or a piece of paper hanging on a wall might lead to counter-intuitive effects. 
Suddenly, those who are important should be (counter-intuitively) drawn at the bottom, whereas we are accustomed to thinking that "the top" communicates importance.

8 We started this warm-up exercise by asking the participants to think about the windows in their homes and tell us everything that came to mind about them. There was a large range of answers among the participants, some simply naming the number of windows and others giving various associations (e.g., stories about their neighbors or daily life). For participants with very little experience in thinking aloud, we added a second warm-up exercise asking for light sources within their home. 9 Several studies have shown how differently participants interpret and understand name generators (e.g. Bailey \& Marsden, 1999; Hollstein, 2002; Bearman \& Parigi, 2004). However, the focus of this article is the visual tools for data collection. Different interpretations and meanings of closeness will only be mentioned when they are related to the visual interface.

10 Participants were recruited via online and offline ads (university research lab, eBay classifieds, and municipal bulletin board). In order to achieve even groups following our experimental design, we sent a brief questionnaire on sociodemographic data to all those interested in participating. With that, we were able to sample participants into the 18 sociodemographic groups until each of the groups was complete.

11 Migration background refers here to the fact that at least one parent was not born in Germany.

12 Because of the small sample sizes, we calculate the significance of differences of average numbers in this article with the Wilcoxon tests (also known as Mann-Whitney test) in R with the command wilcox.test.

13 It is worth noting that the high standard deviations for the number of alters are artifacts that can be created by a single upper outlier when the number of participants is small.

14 In the quantitative analyses, we treat every node (actor) from the map as an alter.

15 In later parts of the interview, when participants reflected on the tools (retrospective thinking aloud), participants elicited on average 0.3 (SD 0.9, range 0-3) further alters. Here, we did not find differences among tools.

16 When network maps with distinct stimuli are used, three circles have been shown to be practical (as in Antonucci, 1986). A variant of Antonucci's diagram is applied by Bernardi et al., 2014, who use six circles, subdividing each of the three circles. For most of our respondents, the three segments worked fine. However, one participant who used the concentric circles allocated alters outside the circles, thus creating a fourth category (cf. also Straus, 2002). Similarly, in an empirical study, re-analyzing the number of alters in tens of different network studies, Zhou et al. (2005) found four discrete hierarchical groups of alters, from very strong to very weak ties.

17 In this experimental study, we stratified the sample according to sociodemographic characteristics that are known to have an impact on the size and composition of personal networks (age, gender, and socioeconomic status). We consider the fact that our results regarding differences in network size among sociodemographic groups are generally in line with findings on personal networks (e.g. Antonucci et al., 2004; McPherson et al., 2006; Wrzus et al., 2013) as an indicator of the quality of the sample-a sample, which is not limited to a specific group (e.g. undergraduates) but covers a broad range of sociodemographic characteristics in the general population.

18 This aspect was stressed by an anonymous reviewer. A solution could be to customize the shape of the funnel tool so that it looks like a segment of a pie chart. Then, the idea of an "angle" or "perspective" could be kept while avoiding ego-centralization as in the concentric circles diagram.

19 We asked the respondents (for standardized tools) to write the initials of their alters on little "sticky dots" and put them on the map. For the free design, we did not give any suggestions. A few participants felt uncomfortable telling alters names or initials and preferred to anonymize them right away by saying "this person," "he/she," or using role relations (e.g. "my friend") (cf. Ryan et al., 2014; Dobbie et al., 2018). Despite having detailed information (written and verbal) on data privacy, it is important to emphasize this issue at the beginning of the interview to give interviewees reliability of expectations. While anonymizing alters might increase data accuracy, it can become a major obstacle in longitudinal studies when single alters over time need to be traced.

20 For instance, adding segments (such as circles) transforms the hard task of comparing every actor with every other actor in terms of who is closer to ego (quadratic complexity) into a categorical (and linear complex) exercise.

21 Since we set up a thinking-aloud approach and participants (independently from the tool they used) participated in this to differing extents, we could not draw conclusions regarding the overall efficiency of each tool.

\section{References}

Antonucci, T. C. (1986). Hierarchical mapping technique. Generations: Journal of the American Society on Aging, 10(4), 10-12. Antonucci, T., Akiyama, H., \& Takahashi, K. (2004). Attachment and close relationships across the life span. Attachment \& Human Development, 6(4), 353-370.

Bailey, S., \& Marsden, P. V. (1999). Interpretation and interview context: Examining the General Social Survey name generator using cognitive methods. Social Networks, 21, 287-309.

Bearman, P., \& Parigi, P. (2004). Cloning headless frogs and other important matters: Conversation topics and network structure. Social Forces, 83(4), 535-557.

Bell, D. C., Belli-McQueen, B., \& Haider, A. (2007). Partner naming and forgetting: Recall of network members. Social Networks, 29(2), 279-299. 
Bellotti, E. (2016). Qualitative methods and visualizations in the study of friendship networks. Sociological Research Online, 21(2), 1-19.

Bernard, H. R., Killworth, P., \& Sailer, L. (1981). Summary of research on informant accuracy in network data and the reverse small world problem. Connections, 4(2), 11-25.

Bernardi, L., Keim, S., \& Klärner, A. (2014). Social networks, social influence, and fertility in Germany: A mixed-method research design. In S. Dominguez, \& B. Hollstein (Eds.), Mixed methods social networks research (pp. 121-152). New York, NY: Cambridge University Press.

Bernardi, L., Keim, S., \& von der Lippe, H. (2007). Social influence on fertility. A comparative mixed methods study in eastern and western Germany. Journal of Mixed Methods Research, 1(1), 23-47.

Bilecen, B. (2016). A personal network approach in mixed-methods design to investigate trans-national social protection. International Review of Social Research, 6(4), 233-244.

Brashears, M. E., \& Quintane, E. (2015). The microstructures of network recall: How social networks are encoded and represented in human memory. Social Networks, 41, 113-126.

Brewer, D. D. (1995). The social structural basis of the organization of persons in memory. Human Nature, 6(4), $379-403$.

Brewer, D. D. (2000). Forgetting in the recall-based elicitation of personal and social networks. Social Networks, 22(1), 29-43.

Brewer, D. D., Rinaldi, G., Mogoutov, A., \& Valente, T. W. (2005). A quantitative review of associative patterns in the recall of persons. Journal of Social Structure, 6(1). Retrieved from https://www.cmu.edu/joss/content/articles/volume6/ Brewer/index_new.html.

Carrasco, J. A., Hogan, B., Wellman, B., \& Miller, E. J. (2008). Collecting social network data to study social activity-travel behavior: An egocentric approach. Environment and Planning B: Planning and Design, 35(6), 961-980.

Coates, D. L. (1985). Adolescent Social record, your social map, social network record. In R. L. Johanes (Eds.), Handbook of tests and measurements for black populations (pp. 269-285). Hampton: Cobb \& Henry Publishers.

Coromina, L., \& Coenders, G. (2006). Reliability and validity of egocentered network data collected via web: A meta-analysis of multilevel multitrait multimethod studies. Social Networks, 28(3), 209-231.

Dobbie, F., Reith, G., \& McConville, S. (2018). Utilising social network research in the qualitative exploration of gamblers' social relationships. Qualitative Research, 18(2), 207-223.

Eddens, K., \& Fagan, J. M. (2018). Comparing nascent approaches for gathering alter-tie data for egocentric studies. Social Networks, 55, 130-141.

Ericsson, K. A., \& Simon, H. A. (1993). Protocol analysis: Verbal reports as data. Cambridge, MA: MIT Press.

Fagan, J. M. \& Eddens, K. S. (2015). OpenEddi: A Network Data Collection Tool. Version 0.3, Lexington, KY: Flaming Fox, LLC.

Feld, S. L. (1981). The focused organization of social ties. American Journal of Sociology, 86(5), 1015-1035.

Gamper, M., Schönhuth, M., \& Kronenwett, M. (2012). Bringing qualitative and quantitative data together: Collecting network data with the help of software tool VennMaker. In M. Safar, \& K. Mahdi (Eds.), Social networking and community behavior modeling: Qualitative and quantitative measures (pp. 193-213). Hershey: IGI Global.

Haselmair, R., Pirker, H., Kuhn, E., \& Vogl, C. R. (2014). Personal networks: A tool for gaining insight into the transmission of knowledge about food and medicinal plants among Tyrolean (Austrian) migrants in Australia, Brazil and Peru. Journal of Ethnobiology and Ethnomedicine, 10(1). doi:10.1186/1746-4269-10-1.

Häussling, R. (2014). A network analytical four-level concept for an interpretation of social interaction in terms of structure and agency. In S. Dominguez, \& B. Hollstein (Eds.), Mixed methods social networks research: Design and applications (pp. 121-177). New York: Cambridge University Press.

Hennig, M., Brandes, U., Pfeffer, J., \& Mergel, I. (2012). Studying social networks: A guide to empirical research. Frankfurt \& New York: Campus Verlag.

Hepp, A., Roitsch, C., \& Berg, M. (2016). Investigating communication networks contextually: Qualitative network analysis as cross-media research. Mediekultur: Journal of Media and Communication Research, 32(60), 87-106.

Hersberger, J. (2003). A qualitative approach to examining information transfer via social networks among homeless populations. The New Review of Information Behaviour Research, 4(1), 95-108.

Herz, A., \& Petermann, S. (2017). Beyond interviewer effects in the standardized measurement of egocentric networks. Social Networks, 50, 70-82.

Herz, A., Peters, L., \& Truschkat, I. (2015). How to do qualitative structural analysis: The qualitative interpretation of network maps and narrative interviews. Forum Qualitative Sozialforschung/Forum Qualitative Social Research, 16(1), Art. 9. Retrieved from http://nbn-resolving.de/urn:nbn:de:0114-fqs150190.

Hogan, B., Carrasco, J. A., \& Wellman, B. (2007). Visualizing personal networks: Working with participant-aided sociograms. Field Methods, 19(2), 116-144.

Hogan, B., Melville, J. R., Phillips II, G. L., Janulis, P., Contractor, N., Mustanski, B. S., \& Birkett, M. (2016). Evaluating the paper-to-screen translation of participant-aided sociograms with high-risk participants. In Proceedings of the $2016 \mathrm{CHI}$ conference on human factors in computing systems - CHI'16 (pp. 5360-5371).

Hollstein, B. (2002). Soziale Netzwerke nach der Verwitwung. Wiesbaden: VS Verlag. 
Hollstein, B. (2011). Qualitative Approaches. In J. Scott, \& P. J. Carrington (Eds.), The SAGE handbook of social network analysis (pp. 404-417). London/New Dehli: SAGE.

Hollstein, B., Behrmann, L., \& Pfeffer, J. (2013). Touchscreen-gesteuerte Instrumente zur Erhebung egozentrierter Netzwerke. In M. Schönhuth, M. Gamper, M. Kronenwett, \& M. Stark (Eds.) Visuelle Netzwerkforschung: Qualitative, quantitative und partizipative Zugänge (pp. 121-136). Bielefeld: Transcript.

Hollstein, B., \& Pfeffer, J. (2010). Netzwerkkarten als Instrument zur Erhebung egozentrierter Netzwerke. In H.-G. Soeffner (Ed.), Unsichere Zeiten. Verhandlungen des 34. Kongress der Deutschen Gesellschaft für Soziologie, 6.-10. Oktober 2008, Jena (pp. 1-13). Frankfurt am Main: Campus.

Kahn, R. L., \& Antonucci, T. C. (1980). Convoys over the life course: Attachment, roles, and social support. In P. B. Baltes, \& G. B. Orville (Eds.), Life-span development and behavior (pp. 253-286). New York: Academic Press.

Keupp, H., Ahbe, T., Gmür, W., Höfer, R., Mitzscherlich, B., Kraus, W., \& Straus, F. (1997). Identitätskonstruktionen. Das Patchwork der Identitäten in der Spätmoderne. Reinbek: Rowohlt.

Kuhns, L. M., Birkett, M., Mustanski, B., Muth, S. Q., Latkin, C., Ortiz-Estes, I., \& Garofalo, R. (2015). Methods for collection of participant-aided sociograms for the study of social, sexual and substance-using networks among young men who have sex with men. Connections, 35(1) doi:10.1186/1746-4269-10-1.

Lang, F. L., \& Carstensen, L. L. (1994). Close emotional relationships in late life: Further support for proactive aging in the social domain. Psychology and Aging, 9(2), 315-324.

Levin, D. Z., Walter, J., \& Murnighan, J. K. (2011). Dormant ties: The value of reconnecting. Organization Science, 22(4), 923-939.

Marsden, P. V. (2011). Survey methods for network data. In J. S. Scott, \& P. J. Carrington (Eds.), The sage handbook of social network analysis (pp. 370-386). Thousand Oaks, CA: Sage.

Marsden, P. V., \& Campbell, K. E. (1984). Measuring tie strength. Social forces, 63(2), 482-501.

McCarty, C., Molina, J. L., Aguilar, C., \& Rota, L. (2007). A comparison of social network mapping and personal network visualization. Field Methods, 19(2), 145-162.

McPherson, M., Smith-Lovin, L., \& Brashears, M. E. (2006). Social isolation in America: Changes in core discussion networks over two decades. American Sociological Review, 71(3), 353-375.

Molina, J. L., Maya-Jariego, I., \& McCarty, C. (2014). Giving meaning to social networks: Methodology for conducting and analyzing interviews based on personal network visualizations. In S. Domínguez, \& B. Hollstein (Eds.), Mixed methods social networks research: Design and applications (pp. 305-335). New York, NY: Cambridge University Press.

Pahl, R., \& Spencer, L. (2004). Capturing personal communities. In C. Phillipson, G. Allan, \& D. Morgan (Eds.), Social networks and social exclusion (pp. 72-96). Aldershot: Ashgate.

Perry, B., Pescosolido, B., \& Borgatti, S. (2018). Egocentric network analysis: Foundations, methods, and models. Cambridge: Cambridge University Press.

Pettigrew, K. E. (1997). The role of community health nurses in providing information and referral to the elderly: A study based on social network theory. London: University of Western Ontario.

Reyes, C. (2016). Eliciting data on social relationships: The use of hand-drawn network maps in tracing the perception of digitally mediated social ties. International Review of Social Research, 6(4), 256-268.

Ryan, L., \& D’Angelo, A. (2018). Changing times: Migrants' social network analysis and the challenges of longitudinal research. Social Networks, 53, 148-158.

Ryan, L., Mulholland, J., \& Agoston, A. (2014). Talking ties: Reflecting on network visualisation and qualitative interviewing. Sociological Research Online, 19(2), 1-12.

Samuelsson, M., Thernlund, G., \& Ringström, J. (1996). Using the five field map to describe the social network of children: A methodological study. International Journal of Behavioral Development, 19(2), 327-345.

Scheibelhofer, E. (2011). Potential of qualitative network analysis in migration studies- reflections based on an empirical analysis of young researchers' mobility aspirations. Migration Letters, 8(2), 111-120.

Schönhuth, M., Gamper, M., Kronenwett, M., \& Stark, M. (Eds.). (2013). Visuelle Netzwerkforschung: Qualitative, quantitative und partizipative Zugänge. Bielefeld: Transcript.

Straus, F. (1995). Egonet QF. Ein Manual zur egozentrierten Netzwerkanalyse für die qualitative Forschung. Ms.

Straus, F. (2002). Netzwerkanalysen. Gemeindepsychologische Perspektiven für Forschung und Praxis. Wiesbaden: Deutscher Universitätsverlag.

Todd, D. M. (1980). Social networks, psychosocial adaptation, and preventive/developmental interventions: The support development workshop. Paper presented at a meeting of the American Psychological Association, Montreal, Canada, September 2.

Tracy, M. E., \& Whittaker, J. K. (1990). The social network map: Assessing social support in clinical practice. Families in Society, 71(8), 461-470.

Tubaro, P., Ryan, L., \& D’Angelo, A. (2016). The visual sociogram in qualitative and mixed-methods research. Sociological Research Online, 21(2), 1-18.

Von der Lippe, H., \& Gamper, M. (2017). Drawing or tabulating ego-centered networks? A mixed-methods comparison of questionnaire vs. visualization-based data collection. International Journal of Social Research Methodology, 20(5), 425-441. 
Wagner, K. D., Syvertsen, J. L., Verdugo, S. R., Molina, J. L., \& Strathdee, S. A. (2018). A mixed methods study of the social support networks of female sex workers and their primary noncommercial male partners in Tijuana, Mexico. Journal of Mixed Methods Research, 12(4), 437-457.

Wagner, M., Schütze, Y., \& Lang, F. R. (1999). Social relationships in old age. In B. Baltes, \& K. U. Mayer (Eds.), The Berlin Aging Study. Aging from 70 to 100 (pp. 282-301). New York: Cambridge University Press.

Wrzus, C., Hänel, M., Wagner, J., \& Neyer, F. J. (2013). Social network changes and life events across the life span: A metaanalysis. Psychological Bulletin, 139(1), 53-80.

Zhou, W. X., Sornette, D., Hill, R. A., \& Dunbar, R. I. (2005). Discrete hierarchical organization of social group sizes. Proceedings of the Royal Society B: Biological Sciences, 272(1561), 439-444.

Cite this article: Hollstein B., Töpfer T., and Pfeffer J. (2020). Collecting egocentric network data with visual tools: A comparative study. Network Science 8, 223-250. https://doi.org/10.1017/nws.2020.4 